\title{
28 Research Square \\ Identification of Novel Biomarkers and Targets for Natural Products in Inhibiting Prostate Cancer
}

\author{
Wenji Li ( $\nabla$ wenji_li@yzu.edu.cn ) \\ Yangzhou University https://orcid.org/0000-0003-0406-9489 \\ Wei Xu \\ Yangzhou University \\ Kai Sun \\ Yangzhou University \\ Fujun Wang \\ Yangzhou University \\ Tin Wui Wong \\ Universiti Teknologi MARA
}

Primary research

Keywords: Prostate Cancer, curcumin, paclitaxel, ursolic acid, network pharmacology, molecular docking

Posted Date: October 1st, 2021

DOI: https://doi.org/10.21203/rs.3.rs-889442/v1

License: (c) (i) This work is licensed under a Creative Commons Attribution 4.0 International License.

Read Full License 


\section{Abstract}

Background: Prostate cancer ( $\mathrm{PCa}$ ) is a common urinary system malignancy. The lack of specific and sensitive biomarkers for the diagnosis and prognosis of PCa makes it important to seek alternatives. Meanwhile, targeted PCa inhibitors are limited. Natural products that potentially target PCa may offer a useful approach.

Methods: Expression profile datasets about PCa from GEO were analyzed. Core differential genes were identified by String and Cytoscape. GEPIA and HPA were utilized to further validate the key genes. The targets of natural products were obtained from the Drugbank, Therapeutic Target Database, BindingDB, PubChem, and chEMBL databases, and PCa therapeutic targets were generated from the GeneCards, OMIM, and PharmGkb databases. Cytoscape was also used to screen the core modules and disease-drug targets. Construction of molecular docking models of drug-core targets was performed by Autodock to confirm the accuracy of the targets.

Results: Four identified biomarkers, CENPF, TPX2, TK1 and CCNB1 were verified by HPA. Five novel PCa biomarkers, RRM2, UBE2C, TOP2A, BIRC5 and ZWINT were also identified. All the nine markers indicated poor prognosis for PCa patients were verified by GEPIA. PCa carcinogenesis is found to be mainly associated with hepatic fibrosis pathway, ILK signaling, NRF2-mediated oxidative stress response and many others. Four key PCa targets for curcumin (EP300, RELA, EGFR, NFKB1), seven for taxol (PTEN, EGFR, ERBB2, TP53, KRAS, AR, AKT1) and two for ursolic acid (GSK3B, RELA) were identified by Cytoscape combined KEGG and verified by Autodock.

Conclusions: The novel identified biomarkers in our study would be valuable for the diagnosis and prognosis of PCa. Key targets of curcumin, paclitaxel, and ursolic acid in PCa could lay a solid foundation for precise treatment and molecularly targeted therapy for PCa.

\section{Background}

The incidence of prostate cancer (PCa) is on the rise globally(1). The prevalence of PCa and associated deaths in China has steeply risen in recent years due to rising living standards, changing diets and an aging population(2). Prostate-specific antigen (PSA) is the most common marker used to identify an increased risk of PCa, but PSA level is not a highly accurate indicator and can be influenced by many other factors such as alcohol consumption and inflammation(3). According to the Chinese expert consensus on genomic testing of PCa patients (2020 edition) and Role of Genetic Testing for Inherited PCa Risk:Philadelphia PCa Consensus Conference 2017, the number of biomarkers that can be used in early diagnosis of PCa is relatively small, and their specificity and sensitivity are $\operatorname{low}(4,5)$. Therefore, it is particularly important to screen novel, highly specific and sensitive biomarkers for the diagnosis and prognosis of PCa.

Common treatment options for PCa include surgical resection, radiotherapy(6), endocrine therapy, and immunotherapy(7), however the number of adverse side effects and frequent progression to refractory 
PCa often have a significant impact on the health and quality of life of PCa patients(8). Molecular targeted therapy (MTT) refers to the inhibition of tumor growth and development by interfering with specific molecules that increase proliferation and migration of tumor cells(9). Currently there are only a few molecularly targeted drugs for $\mathrm{PCa}(10)$, and since none of them are very specific, it is essential to identify alternatives as well.

Natural products are certain chemicals obtained from naturally growing organisms(11). Curcumin is extracted from turmeric and is an important active ingredient in traditional Chinese medicine. Curcumin has good antioxidant, anti-pathogenic microorganism, anti-rheumatism and anti-tumor activities, and can also improve the function of the immune, cardiovascular, digestive and nervous systems(12). Paclitaxel is a natural secondary metabolite isolated and purified from the bark of the Pacific yew(13). Paclitaxel and its synthetic variants are known to have anti-cancer properties and have been used extensively in the clinic, but their use for treatment of PCa is less frequent and has been described primarily in laboratory studies(14). Ursolic acid is extracted from fruit rind and has sedative and anti-inflammatory, antibacterial, anti-diabetic, anti-ulcer effects and antioxidant properties(15). Recent studies have shown that all the three natural products have significant inhibitory effects on cancer, but their mechanisms of action and targets in PCa have not been thoroughly investigated. Therefore, this study aimed to identify targets of these three natural products in PCa in order to provide theoretical support for their use in MTT of PCa.

In our study, genes associated with the diagnosis and prognosis of PCa were screened using bioinformatics software, in the meanwhile key targets of natural products that act on PCa were analyzed as well. A molecular model of natural product docking with PCa targets was constructed based on network pharmacology to confirm the accuracy of the PCa-natural products targets. This study would provide solid support for the development of MTT of PCa.

\section{Methods}

\subsection{Raw data selection}

Differences in gene expression between PCa and normal tissues were analyzed using the GEO module on the Assistant for Clinical Bioinformatics (Aclbi) website (www.Aclbi.com), by selecting datasets in the Dataset Screening and Differential Gene Analysis sub-modules. Gene expression data for both GSE3325(16) and GSE46602(17) were based on the GPL570 platform (Affymetrix Human Genome U133 Plus 2.0 Array). The GSE3325 dataset contains information from 6 benign prostate tissues and $13 \mathrm{PCa}$ tissues; and the GSE46602 dataset contains information from 14 benign prostate tissues and $36 \mathrm{PCa}$ tissues (Additional file : Table. S1). (The GEO data in Aclbi is updated every 3 months and the latest updated version on May 17, 2021 was utilized.)

\subsection{Data processing and screening of differentially expressed genes(DEGs)}


The selected data were processed using the GEO-Differential Gene Module of the Aclbi. The website is based on $\mathrm{R}$ software (v4.0.3) core to run automatic data processing, including normalization of data, debatching effects and other related processing to ensure validity and accuracy. The data obtained from the Aclbi were further processed using Ingenuity Pathways Analysis (IPA, Qiagen 2021) software to improve the accuracy of the data. The data obtained from the Aclbi for GEO were analyzed using the CoreAnalysis module in the IPA software with a cutoff value of $|\operatorname{logFC}|>1, p<0.05$ to obtain the final DEGs .

\subsection{GO enrichment analysis and IPA pathway analysis}

The Database for Annotation, Visualization and Integrated Discovery (DAVID) database (https://david.ncifcrf.gov/)(18) was used for analysis of DEGs by Gene Ontology (GO)(19) enrichment methods. GO enrichment analysis focuses on describing the biological processes (BPs), cellular components (CCs) and molecular functions (MFs) associated with DEGs. The pathway analysis of DEGs was performed by IPA. A cutoff value of $p<0.05$ was used. The hiplot database (https://hiplot.com.cn), a comprehensive web platform for scientific data visualization, was used to visualize the enrichment results.

\subsection{Protein-Protein Interactions (PPI) network construction, core genes and modules screening}

Interrelationships between the DEGs were constructed using the String(20) database (https://www.string$\mathrm{db} . o r g /$ ) (version 11.5), with interrelationship scores calculated from text mining, experiments, databases, co-expression neighborhood, gene fusion, and co-occurrence, while hiding unrelated nodes in the network

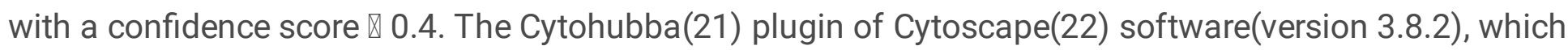
is based on the maximal clique centrality algorithm, was used to identify the connected genes most closely associated with the development of PCa. The top 30 scored genes were selected. In addition, the molecular complex detection (MCODE)(23) app in Cytoscape was used to check modules of the PPI network (degree cutoff $=2$, max. Depth $=100$, $k$-core $=2$, and node score cutoff $=0.2$ ). The enrichment analysis of core modules was performed by the DAVID and Hiplot.

\subsection{Validation of the expression of core genes and survival analysis}

The Gene Expression Profiling Interactive Analysis (24) (GEPIA; http://gepia.cancer-pku.cn/) database, launched in 2017 and now cited in a large number of articles, is an efficient, accurate and convenient database for gene expression analysis based on tumor and normal samples from the Cancer Genome Atlas (TCGA) and the Genotype-Tissue Expression (GTEx) databases. GEPIA was used to further validate expression of core genes screened by Cytoscape, by setting $|\log F C|>1$ and $p<0.01$ as the cutoff value, with matched tumor-normal tissue data from the TCGA and GTEx databases respectively. In addition, the survival analysis of core genes was performed by GEPIA database as well. The protein expression of key genes in PCa and normal tissues was analyzed by the human protein atlas(HPA, 
https://www.proteinatlas.org/ ) (Version: 20.1)(25). The pan-cancer analysis for core genes was performed in Timer2(http://timer.cistrome.org/) database(26).

\subsection{PCa treatment targets}

Validated PCa targets were identified in the GeneCards(27) (https://www.genecards.org/), Online Mendelian Inheritance in Man(28) (OMIM,https://omim.org/) and PharmGkb(29)

(https://www.pharmgkb.org/) databases. Targets with relevance scores > 1 in the GeneCards database were considered to be significant. The targets retrieved from the three databases were combined with the core differential genes while duplicate values were removed, resulting in the set of therapeutic targets for PCa.

\subsection{Natural product targets}

The DrugBank(30) (https://go.drugbank.com/), Therapeutic Target Database (TTD)(31) (http://db.idrblab.net/ttd/), BindingDB(32) (http://www.bindingdb.org/bind/index.jsp), PubChem(33) (https://pubchem.ncbi.nlm.nih.gov/), and ChEMBL(34) (https://www.ebi.ac.uk/chembl/) databases were used to identify the targets of curcumin/paclitaxel/ursolic acid. The targets identified in the five databases were combined while duplicate values were removed.

\subsection{Disease-natural product targets}

Intersections of the PCa and curcumin/paclitaxel/ursolic acid targets were identified in Excel following Duilio's method(35), and the resulting targets were designated pharmacodynamic targets of curcumin/paclitaxel/ursolic acid in PCa.

\subsection{Construction of PPI networks of PCa-natural product targets and screening of core modules}

Information about PCa-natural products was imported into the String database to construct a network of interactions between targets, and interactions with a confidence score of 0.4 or more were retained. The inter-target interaction scores were also imported into Cytoscape software for core module screening by using the CytoNCA(36) plugin, and the median of the Betweenness Centrality (BC), Closeness Centrality (CLC), Degree Centrality (DC), Eigenvector Centrality (EC), Local Average Connectivity-based method (LAC) and Network Centrality (NC) scores was used as the basis for screening of genes to obtain their core motifs.

\subsection{GO and KEGG enrichment analysis of disease-natural product targets}

DAVID database was used to provide data for the GO and KEGG analysis of disease-natural product targets, when the $p$-value was set as 0.05 or less. The Hiplot online database was used for enrichment analysis of disease-natural product targets for mapping with a p-value $<0.05$ and q-value $<0.05$.

2.11 Construction of molecular docking models 
Protein receptors were identified based on the KEGG enrichment results of the disease-natural product targets. The small-molecule ligands were curcumin/paclitaxel/ursolic acid. 2D structures of curcumin/paclitaxel/ursolic acid were downloaded from the PubChem database and converted into 3D structures by ChemOffice software(version 14.0.0.117), and the structure was adjusted to minimize the binding energy, where the minimum RMS gradient $=0.01$. Protein receptor $3 \mathrm{D}$ structures were obtained from the Protein Data Bank(37) (PDB, https://www1.rcsb.org/). In PyMoL (version 2.4.0) software(38), the water molecules and the original small-molecule ligand were removed from the 3D structure. The 3D protein structure was hydrogenated using AutoDockTools software(version 1.5.6)(39), and a grid box was added to the protein structure with different reference values depending on the protein receptors.

Molecular docking was performed in AutodockVina by setting the energy range to 5. Targets with binding energy lower than $-5 \mathrm{kcal} / \mathrm{mol}$ are retained, and the lower the binding energy, the affinity between small molecules and targets. The resulting models were displayed using PyMoL software.

\section{Results}

In IPA, $|\log F C|>1$ and a p-value $<0.05$ were set as the cutoff value to obtain DEGs from the GEO module. A total of 582 DEGs were obtained after analysis of the GEO dataset, of which 201 were up-regulated and 381 were down-regulated(Fig. 1,Table 1). 
Table 1

GEO differentially expressed genes

\begin{tabular}{|c|c|}
\hline Category & Genes \\
\hline $\begin{array}{l}\text { Up- } \\
\text { regulated } \\
\text { DEGs } \\
\text { names }\end{array}$ & 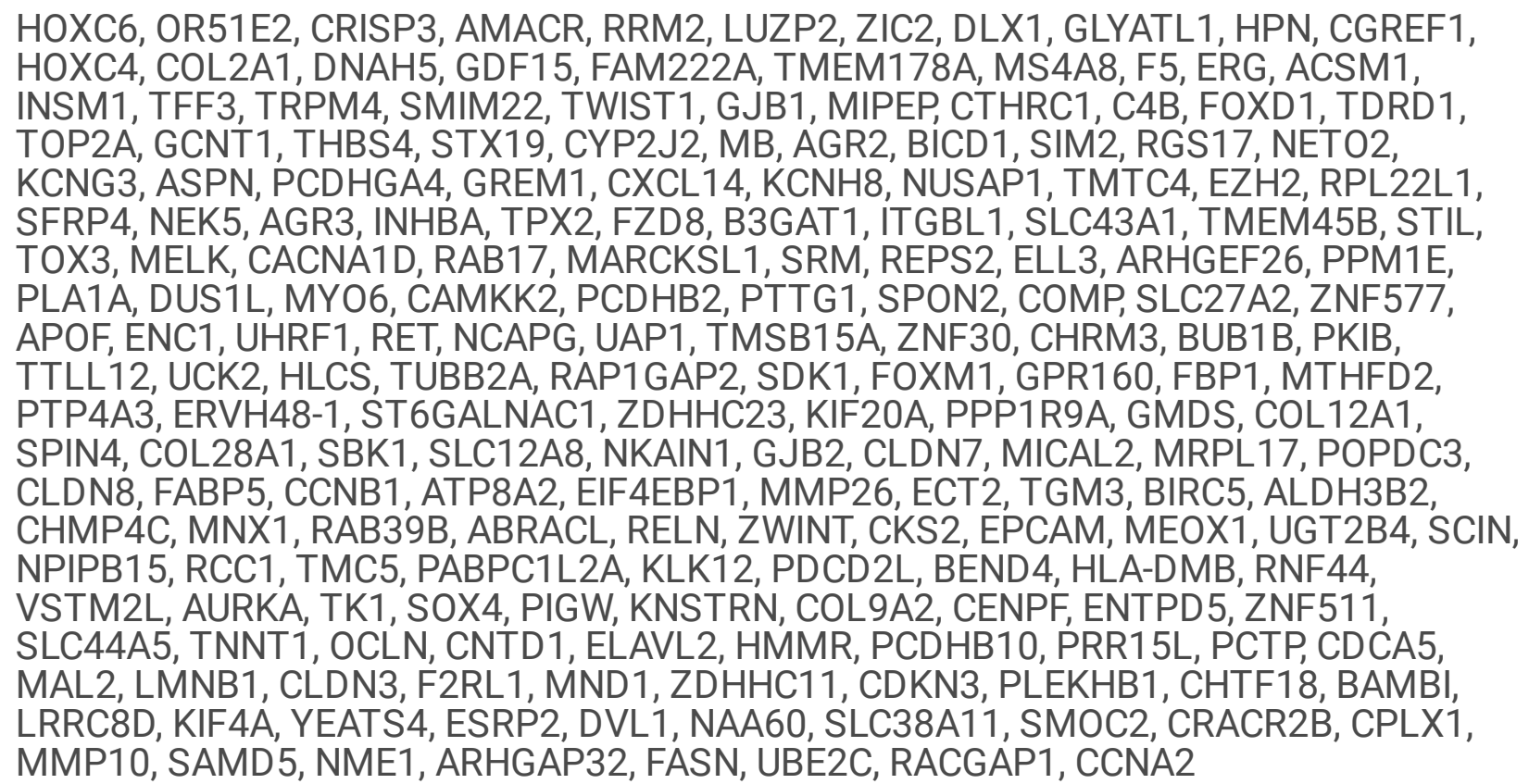 \\
\hline
\end{tabular}




\begin{tabular}{|c|c|}
\hline Category & Genes \\
\hline $\begin{array}{l}\text { Down- } \\
\text { regulated } \\
\text { DEGs } \\
\text { names }\end{array}$ & 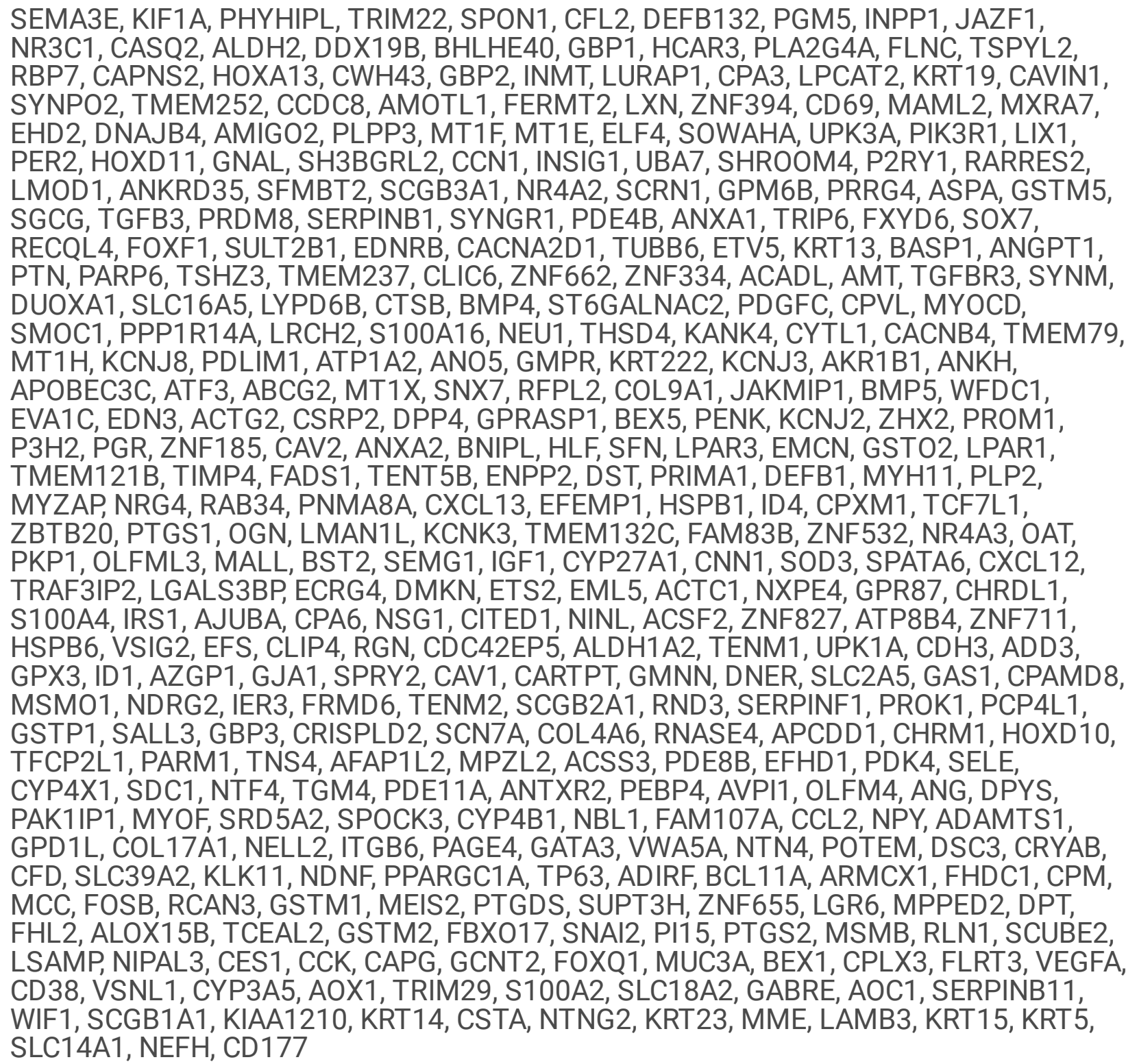 \\
\hline
\end{tabular}

\subsection{GO and IPA pathway analysis}

After analysis of the information in the DAVID database, GO analysis revealed that biological processes BPs were enriched in muscle system process, cell junction assembly, epithelial cell proliferation, muscle contraction, extracellular matrix organization, regulation of epithelial cell proliferation, extracellular structure organization, regulation of actin filament-based process, muscle cell differentiation, and others. (Fig. 2a). MFs were enriched in receptor ligand activity, signaling receptor activator activity, sulfur compound binding, actin binding, enzyme inhibitor activity, glycosaminoglycan binding, heparin binding, tubulin binding, extracellular matrix structural constituent and others.(Fig. 2b). CCs were enriched in collagen-containing extracellular matrix, cell-cell junction, cell-substrate junction, focal adhesion, membrane raft, membrane microdomain, membrane region, contractile fiber, myofibril, microtubule, sarcomere, cell leading edge, apical part of cell (Fig. 2c). IPA pathway analysis indicated the major pathways associated with carcinogenesis of PCa includes: hepatic fibrosis/hepatic stellate cell 
activation, ILK signaling, xenobiotic metabolism PXR signaling pathway, xenobiotic metabolism AHR signaling pathway, LPS/IL-1 mediated inhibition of RXR function, xenobiotic metabolism CAR signaling pathway, SPINK1 pancreatic cancer pathway, aryl hydrocarbon receptor signaling, NRF2-mediated oxidative stress response, MSP-RON signaling in cancer cells pathway, osteoarthritis pathway, agranulocyte adhesion and diapedesis, nicotine degradation II, human embryonic stem cell pluripotency, amyotrophic lateral sclerosis signaling, MSP-RON signaling pathway, WNT/ $\beta$-catenin signaling, endocannabinoid cancer inhibition pathway and others.(Fig. 2d).

\subsection{PPI network construction, core genes and modules screening}

As indicated in Fig. 3a, by using the String database, we constructed a protein relationship network graph for DEGs, in which there were 582 nodes and 1869 edges after removing the unlinked nodes. Thirty genes with the highest scores, namely NCAPG, UBE2C, AURKA, BUB1B, TPX2, CENPF, MELK, RACGAP1, NUSAP1, HMMR, TOP2A, CCNA2, CCNB1, ZWINT, RRM2, CDKN3, BIRC5, CDCA5, KIF20A, KIF4A, FOXM1, PTTG1, ECT2, CKS2, EZH2, TK1, UHRF1, MND1, GMNN, and STIL, were obtained using the Cytohubba plugin in Cytoscape software (Fig. 3b). Two modules with the highest scores were screened. Module 1(Fig. 3c, 3e), with 31 nodes and 434 edges, scored 28.933, in the GO analysis revealed that BPs were enriched in nuclear division, organelle fission, mitotic nuclear division, chromosome segregation, nuclear chromosome segregation, sister chromatid segregation, mitotic sister chromatid segregation, etc, MFs were enriched in microtubule binding, tubulin binding, protein kinase regulator activity, kinase regulator activity, etc, and CCs were enriched in spindle, chromosomal region, chromosome-centromeric region, condensed chromosome, condensed chromosome-centromeric region, midbody, microtubule, etc. The KEGG pathway analysis was mainly concentrated in cell cycle, progesterone-mediated oocyte maturation, oocyte meiosis, cellular senescence, human T-cell leukemia virus 1 infection, etc. Module 2(Fig. 3d, 3f), with 15 nodes and 33 edges, scored 4.714, in the GO analysis revealed that BPs were enriched in muscle system process, cell junction assembly, epithelial cell proliferation, muscle contraction, extracellular matrix organization, regulation of epithelial cell proliferation, extracellular structure organization, regulation of actin filament-based process, muscle cell differentiation, etc, MFs were enriched in efflux transmembrane transporter activity, sulfur compound transmembrane transporter activity, E-box binding, amide transmembrane transporter activity, peroxidase activity, etc, and CCs were enriched in membrane raft, membrane microdomain, membrane region, apical plasma membrane, apical part of cell, etc. KEGG analysis indicated no gene enrichment in possible pathways.

\subsection{Validation of core prognosis genes by GEPIA}

The expression levels of the DEGs were further validated in the GEPIA database using a cutoff value of $|\log F C|>1$ and $p<0.01$. Then 9 key candidate genes were screened out, which may be closely related to 
the occurrence and development of PCa, namely UBE2C, TPX2, CENPF, TOP2A, CCNB1, ZWINT, RRM2, BIRC5, and TK1(Fig. 4a). According to GEPIA's disease free survival analysis, all nine genes were closely

related to the poor prognosis of PCa (Fig. 4b). Survival analysis showed that the high-risk values of all validated genes were greater than 1 , indicating that they were all high-risk genes for PCa with poor prognosis outcomes.

\subsection{Validation of key diagnosis genes by HPA}

According to the results of GEPIA expression analysis, these 9 key genes were highly expressed in PCa and low in normal cancer tissues. HPA database, which contains plenty of clinical immunohistochemical (IHC) sample data, can be used as a tool to validate the key diagnosis genes. From HPA analysis, CENPF, TPX2, TK1 and CCNB1 were found to be the most extensively studied diagnosis genes from the 9 candidates(Fig. 5a), which were only expressed in prostate tumor tissues but not in normal prostate tissues. RRM2 was not expressed in both normal and tumor tissues. UBE2C, TOP2A and BIRC5 were expressed in both normal tissues and tumor tissues with no significant difference. ZWINT has no data in this database. After validation by HPA, CENPF, TPX2, TK1 and CCNB1 were found to be more certain in the carcinogenesis of $\mathrm{PCa}$ and can be used as diagnosis biomarkers. The other 5 genes, RRM2, UBE2C, TOP2A, BIRC5 and ZWINT would be potential diagnosis markers for PCa and need further studies to validate. The pan-cancer analysis in Timer2(40) database indicated the expression of all the 9 genes were significantly upregulated in most cancers including PCa, which suggest their potency of being the diagnosis markers of PCa (Fig. 5b).

\subsection{Disease-Drug targets initial identification}

The GeneCards, OMIM and PharmGkb databases were used to search for PCa targets. 10,055 targets were found in the GeneCards database, 403 in the OMIM database and 411 in the PharmGkb database. After combining the analysis with the DEGs and removing duplicate targets, a total of 10,366 targets were identified for PCa (Fig. 6, Additional file: Table. S2).

\subsubsection{PCa-curcumin initial targets}

A total of 141 curcumin targets were found in the DrugBank, TTD, BindingDB, PubChem and chEMBL databases after removing duplicate targets (Additional file: Table. S3), and a total of 114 intersections of the initial PCa and curcumin targets were obtained.(Table 2)

\subsubsection{PCa-paclitaxel initial targets}

After searching the above 5 databases, 121 targets of paclitaxel were obtained after removing duplicate targets (Additional file: Table. S3), and 105 targets were obtained after taking the intersections (Table 2) with PCa targets, which constituted the initial PCa-paclitaxel targets.

\subsubsection{PCa-ursolic acid initial targets}


In the same five databases, a total of 59 targets (Additional file: Table. S3) were obtained after removal of duplicate targets, and 41 initial PCa-ursolic acid targets (Table 2) were obtained from the intersections with PCa targets.

\subsection{Construction of PPI network of secondary disease-drug targets and core motif screening \\ 3.6.1 PCa-curcumin secondary targets}

A PPI network graph of PCa-curcumin targets was constructed from the String database. 109 nodes and 623 edges were obtained in the network graph after removing unconnected nodes (Fig. 7a). The PPI network was analyzed in Cytoscape software by using the CytoNCA plugin, and again using R software. The targets were screened according to the median of the BC, CLC, DC, EC, NC and LAC scores, and targets with scores larger than the median were retained. The core motifs of these targets were calculated. The first screening, calculated by CytoNCA and R software, was based on BC: 55.12646418 ; CLC: 0.397058824; DC: 10; EC: 0.03516328; LAC: 3.75; and NC: 5.047619048. Then we obtained 26 nodes with 180 edge secondary core motifs (Fig. 7b), and the same calculations and filtering were performed for this motif. The second filtering values were based on BC: 10.48737374 ; CLC: 0.684210526 ; DC: 14; EC: 0.175377861; LAC: 8.545454545; and NC: 10.77973138; resulting in a key motif containing 9 nodes and 36 edges (Fig. 7c, 7d). Finally, the secondary core PCa-curcumin targets were identified as STAT3, PTGS2, RELA, PPARG, EGFR, IL1B, NFKB1, SRC and EP300.

\subsubsection{PCa-paclitaxel secondary targets}

A PPI network graph of PCa-paclitaxel targets was constructed from the String database. 100 nodes and 951 edges were obtained after removing unconnected nodes (Fig. 8a). The PPI network was analyzed in Cytoscape software by using the CytoNCA plugin, while the data analyzed by Cytoscape was analyzed again with the $\mathrm{R}$ software. The targets were screened according to the median of the BC, CLC, DC, EC, NC and LAC scores, and all targets with scores larger than the median were retained. The core motifs of these were obtained by calculation. The first screening, calculated by CytoNCA and R software, was based on BC: 40.031635475 ; CLC: 0.4817546765; DC: 16; EC: 0.0590513925; LAC: 9.4142857145 ; and NC: 11.252039625 . Then we obtained a secondary core motif with 28 nodes and 267 edges (Fig. 8b), and the same calculation and filtering were performed again for this motif. The second filtering values were based on BC: 4.953907041; CLC: 0.782773109; DC: 19.5; EC: 0.1917769165; LAC: 15.677777778; and NC: 17.37566557; resulting in a key motif containing 13 nodes and 78 edges (Fig. 8c, 8d). Finally, the secondary core PCa-paclitaxel targets were identified as SRC, ATM, PTEN, CASP3, CDKN2A, NOTCH1, EGFR, ERBB2, TP53, KRAS, AR, AKT1 and BRCA1.

\subsubsection{PCa-ursolic acid secondary targets}

A PPI network graph of PCa-ursolic acid targets was constructed from the String database. The PPI graph with 38 nodes and 78 edges was obtained after removing unconnected nodes (Fig. 9a) The PPI were analyzed in Cytoscape software using the CytoNCA plugin, and the data analyzed by Cytoscape was 
analyzed again with $\mathrm{R}$ software. The targets were screened according to the median of the BC, CLC, DC, $\mathrm{EC}, \mathrm{NC}$ and LAC scores, and all genes with scores larger than the median were retained. The core motifs were obtained by calculation. The targets were screened based on BC: 17.015873015; CLC: 0.359223301; DC: 3; EC: 0.054310229 ; LAC: 1; NC: 1.5833333335 using CytoNCA and R software. The core motifs of 7 nodes and 11 edges were obtained afterward (Fig. 9b, 9c), and the secondary core PCa-ursolic acid targets were identified as PTGS2, PLA2G1B, PPARA, RELA, HIF1A, GSK3B and HDAC1.

\subsection{Functional enrichment results for disease-drug targets}

GO and KEGG enrichment for disease-drug targets was performed using the DAVID database and analyzed and mapped on the Hiplot website to explore the most relevant pathways to the PCa carcinogenesis and to find the PCa pathway highly associated targets.

\subsubsection{Functional analysis of PCa-curcumin targets}

As shown in Fig. 10a, GO analysis revealed that BPs were enriched in positive and negative regulation of transcription from the RNA polymerase II promoter, negative regulation of apoptosis, responses to drugs, negative regulation of transcription, DNA-templates, signal transduction, and oxidation-reduction processes. MFs were enriched in protein binding, zinc ion binding, enzyme binding, ATP binding, and transcription factor binding. CCs were enriched in cytoplasm, nucleus, cytosol, plasma membrane, extracellular exosomes, and nucleoplasm. In terms of KEGG pathway enrichment, the targets were focused on viral carcinogenesis, pathways in cancer, gap junctions, alcoholism, pathogenic Escherichia coli infection, and phagosomes. Based on KEGG pathway enrichment results, 7 targets were enriched in the PCa pathway, namely GSK3B, CREBBP, MDM2, EP300, RELA, EGFR, NFKB1.

\subsubsection{Functional analysis of PCa-paclitaxel targets}

In Fig. 10b, GO analysis revealed that BPs were enriched in positive regulation of transcription from the RNA polymerase II promoter, cell proliferation, positive and negative regulation of apoptosis, positive and negative regulation of transcription, DNA-templates, apoptosis, positive and negative regulation of cell proliferation, protein phosphorylation, response to drugs, microtubule-based processes, peptidyl-tyrosine phosphorylation, protein autophosphorylation, MAPK cascade, and intracellular signal transduction. MFs were enriched in protein binding, ATP binding, enzyme binding, protein kinase activity, protein homodimerization and heterodimerization, identical protein binding, structural constituents of the cytoskeleton, GTPase activity, GTP binding, protein kinase binding, protein tyrosine kinase activity, kinase activity and CCs. In terms of KEGG pathway enrichment, the targets were focused on pathways in cancer, microRNAs in cancer, PI3K-Akt signaling pathway, hepatitis B, gap junction, proteoglycans in cancer, prostate cancer, HTLV-I infection, melanoma, phagosomes, MAPK signaling pathway, pathogenic Escherichia coli infection, focal adhesions, central carbon metabolism in cancer, glioma, pancreatic cancer, ErbB signaling pathway, FOXO signaling pathway, bladder cancer, colorectal cancer, chronic myeloid leukemia, chemical carcinogenesis, viral carcinogenesis, Rap1 signaling pathway, non-small cell lung cancer, metabolism of xenobiotics by cytochrome P450, estrogen signaling pathway, thyroid 
hormone signaling pathway, neurotrophin signaling pathway, regulation of the actin cytoskeleton, and the Ras signaling pathway. Based on KEGG pathway enrichment results, 16 targets were enriched in the PCa pathway, namely PDGFRA, CDKN1A, PTEN, EGFR, PIK3CG, AR, PIK3CA, ERBB2, CDK2, E2F1, BCL2, AKT1, KRAS, RAF1, TP53, FGFR1.

\subsubsection{Functional analysis of PCa-ursolic acid targets}

As shown in Fig. 10c, GO analysis revealed that BPs were enriched in histone $\mathrm{H} 3$ deacetylation, oxidationreduction processes, protein deacetylation, peptidyl-tyrosine dephosphorylation, negative regulation of myotube differentiation, regulation of transcription from the RNA polymerase II promoter in response to hypoxia, negative regulation of the insulin receptor signaling pathway, response to hypoxia, peptidylproline hydroxylation to 4-hydroxy-L-proline, positive regulation of receptor biosynthetic process, histone deacetylation, and inflammatory responses. MFs were enriched in NAD-dependent histone deacetylase activity (H3-K14 specific), histone deacetylase binding, protein deacetylase activity, histone deacetylase activity, protein tyrosine phosphatase activity, transcription factor binding, enzyme binding, peptidylproline 4-dioxygenase activity, NF-kappaB binding, repression of transcription factor binding, and protein kinase binding. CCs were enriched in the histone deacetylase complex, nucleus, cytosol, nucleoplasm, protein complexes, cytoplasm, transcriptional repressor complexes, Golgi apparatus, and extracellular exosomes. In terms of KEGG pathway enrichment, the targets were focused on viral carcinogenesis, insulin resistance, HIF-1 signaling pathway, pathways in cancer, arachidonic acid metabolism, renal cell carcinoma, insulin signaling pathway, galactose metabolism, starch and sucrose metabolism, alcoholism, thyroid hormone signaling pathway, regulation of lipolysis in adipocytes, and steroid hormone biosynthesis. Based on the enrichment results of the KEGG pathway, 3 targets were enriched in the PCa pathway: GSK3B, RD5A2 and RELA.

\subsection{Construction of molecular docking models}

The molecular docking was utilized to determine the tightness of binding between the target proteins and the natural products, which will be used to confirm the final PCa-natural products' targets.

\subsubsection{Curcumin-receptor protein molecular docking models}

Four final PCa-curcumin targets were identified from intersections with the secondary core targets and 7 targets from KEGG pathway enrichment results, namely EP300, RELA, EGFR, and NFKB1. The four targets were used as protein receptors in the molecular docking models with curcumin as the small-molecule ligand to confirm the final PCa-curcumin targets. When EP300 was used as the protein receptor, the spacing of the gird box in the AutodockTools was set to 1 , the number of points in the $x-, y-$, and $z-$ dimensions was set to 40, and the offset values of the Center Grid Box were: $x$ center: -40.097 ; $y$ center: 97.454; and $z$ center: 194.199. Molecular docking was performed using Vina, which resulted in a model located at the center of the active site with the lowest binding energy, where the binding energy was - 6.9 $\mathrm{kcal} / \mathrm{mol}$. PyMoL was used to obtain the model (Fig. 11a). The construction of molecular docking models for the other three genes was completed by using the same method. For RELA, the spacing of the gird box 
in the AutodockTools was set to 1, the number of points in the $x-, y$-, and $z$-dimensions was set to 40 , and the offset values of the Center Grid Box were: $x$ center: 62.502; $y$ center: 11.629; and $z$ center: 37.997. The binding energy of the model was $-6.9 \mathrm{kcal} / \mathrm{mol}$. For EGFR, the spacing of the gird box in AutodockTools was set to 1 , the number of points in the $x-, y$, and $z$-dimensions was set to 40 , and the offset values of the Center Grid Box were: $x$ center: -0.112 ; $y$ center: -0.618 ; and $z$ center: 0.111 . The binding energy of the model was $-5.3 \mathrm{kcal} / \mathrm{mol}$. For NFKB1, the spacing of the gird box in AutodockTools was set to 1, the number of points in $x-, y$-, and $z$-dimensions was set to 40 , and the offset values of the Center Grid Box were: $x$ center: -0.743 ; $y$ center: -3.335 ; and $z$ center: -0.161 . The binding energy of the model was -5.8 $\mathrm{kcal} / \mathrm{mol}$ (Fig. 11b, 11c, 11d). The binding energies of the four targets to curcumin were lower than - 5 $\mathrm{kcal} / \mathrm{mol}$, indicating that the binding was stable and the affinity was strong, so all the four targets were confirmed as the final PCa-curcumin targets .

\subsubsection{Paclitaxel-receptor protein molecular docking models}

Seven final PCa- Paclitaxel targets were found from the intersections with the secondary core targets and 16 targets from pathway enrichment results, namely PTEN, EGFR, ERBB2, TP53, KRAS, AR, and AKT1. These seven targets were used as protein receptors in the molecular docking models with paclitaxel as the small-molecule ligand. When using PTEN as the protein receptor, the spacing of the gird box in AutodockTools was set to 1 , the number of points in the $x-, y-$, and $z$-dimensions was set to 40 , and the offset values of the Center Grid Box were: $x$ center: 36.248; $y$ center: 82.41; and $z$ center: 31.742. The model with the lowest binding energy was obtained using Vina for molecular docking, where the binding energy was $-7.4 \mathrm{kcal} / \mathrm{mol}$. PyMoL was used to obtain the model (Fig. 12a). Construction of molecular docking models for the other six genes was completed using the same method. We set all the values of spacing, number of points in the $x-, y-$, and $z$-dimensions to the same value as PTEN. For EGFR, the offset values of the Center Grid Box were: $x$ center: -0.112 ; $y$ center: -0.618 ; and $z$ center: 0.111 ; the binding energy was $-5.5 \mathrm{kcal} / \mathrm{mol}$. For ERBB2, the offset values of the Center Grid Box were: $x$ center: 0.017; $y$ center: 0.054; and z center: 0.005; the binding energy was $-6.8 \mathrm{kcal} / \mathrm{mol}$. For TP53, the offset values of the Center Grid Box were: $x$ center: -21.948 ; y center: 0.61; and $z$ center: 22.886; the binding energy was $9.2 \mathrm{kcal} / \mathrm{mol}$. For KRAS: the offset values of the Center Grid Box were: $x$ center: $52.69 ; y$ center: 108.574; and $z$ center: 30.638 ; the binding energy was $-9.0 \mathrm{kcal} / \mathrm{mol}$. For AR: the offset values of the Center Grid Box were: $x$ center: 21.912; y center: 5.289; and $z$ center: 10.612 ; the binding energy was $-6.6 \mathrm{kcal} / \mathrm{mol}$. For AKT1: the offset values of the Center Grid Box were: $x$ center: 13.902 ; $y$ center: -0.811 ; and $z$ center: 3.298 ; the binding energy was $-6.9 \mathrm{kcal} / \mathrm{mol}$ (Fig. $12 \mathrm{~b}-\mathrm{g}$ ). The binding energies of the seven targets to paclitaxel were lower than $-5 \mathrm{kcal} / \mathrm{mol}$, indicating that the binding was stable and the affinity was strong, so all the seven targets were confirmed as the final PCa-Paclitaxel targets.

\subsubsection{Ursolic acid-receptor protein molecular docking models}

Two final PCa-ursolic targets were found from the intersections with the secondary core targets and 3 targets from KEGG pathway enrichment results, namely GSK3B and RELA, which were also used as the protein receptors in the molecular docking models when ursolic acid was used as the small-molecule 
ligand. When GSK3B was used as the protein receptor, the spacing of the gird box in AutodockTools was set to 1 , the number of points in the $x$-, $y$-and $z$-dimensions was set to 40 , and the offset values of the Center Grid Box were: $x$ center: 0.296; y center: 3.935 ; and $z$ center: -4.831 . The model with the lowest binding energy was obtained using Vina for molecular docking, where the binding energy was -7.4 $\mathrm{kcal} / \mathrm{mol}$. When RELA was used as the protein receptor, the spacing of the gird box in AutodockTools was set to 1 , the number of points in the $x-y$-and $z$-dimensions was set to 40 , and the offset values of the Center Grid Box were: $x$ center: 62.502; y center: 11.629; and $z$ center: 37.997. The model with the lowest binding energy was obtained after analysis using Vina for molecular docking, where the binding energy was $-8.1 \mathrm{kcal} / \mathrm{mol}$. PyMoL was used to obtain the model (Fig. 13a, 13b). The binding energies of the two targets to ursolic acid were lower than $-5 \mathrm{kcal} / \mathrm{mol}$, indicating that the binding was stable and the affinity was strong, so both targets were confirmed as the final PCa-Ursolic acid targets.

\section{Discussion}

\subsection{PCa biomarkers analysis}

According to HPA results, the 9 PCa associated genes can be classified into 2 groups, the well-studied biomarkers and the relatively less-studied biomarkers. CENPF, TPX2, TK1 and CCNB1 were only expressed in prostate tumor tissues but not in normal prostate tissues by the clinical IHC results (Fig. 5a), while there is no significant difference for the expression of RRM2, UBE2C, TOP2A, BIRC5 and ZWINT in PCa and normal prostate tissues. However, the lack of significant difference between PCa and normal samples may probably due to the relatively limited samples stored in HPA. In addition, from the pancancer analysis, it demonstrates that the expression of all the 9 genes are significantly upregulated in most cancers including PCa, which suggest the value of studying the 9 genes as potential diagnosis markers of PCa (Fig. 5b).

\subsubsection{The well-studied PCa biomarkers}

The identified well-studied PCa diagnosis markers from our bioinformatics study indicate the rationality of the study design.

Mitotic-specific cyclin-B1(CCNB1) played an important stabilization role of mitotic process in the $\mathrm{G} 2$ to $\mathrm{M}$ phase of the cell cycle(41). Through IHC experiments, CCNB1 was found to be overexpressed in PCa specimens, and acted as a prognostic marker of PCa chemotherapy(42). It is found that prostate cancer cells with overexpressed CCNB1 were more sensitive to chemically induced apoptosis(43).

Centromeric protein F (CENPF) is the basic element of the kinetochore complex and plays an important role in the chromosome separation mechanism of mitosis(44). CENPF was confirmed to be a prognostic marker in $\mathrm{PCa}$ and its expression was found up-regulated in more severe PCa patients with higher Gleason score, later pathological stage, and lymph node metastasis(45). 
Thymidine kinase-1 (TK-1) has been extensively studied as a diagnostic biomarker for various tumors including $\mathrm{PCa}(46)$. It was found that the serum level of TK1 was significantly higher in PCa patients than in normal subjects, and the expression levels were significantly different between PCa patients in different stages(47). Increased TK1 in cancer patients after surgery or chemotherapy treatment are associated with a worse prognosis(48).

Targeting protein for Xenopus kinesin-like protein 2 (TPX2) is a microtubule-associated protein that plays an important role in the mechanism of chromosome segregation in mitosis(49). It is a good biomarker for the diagnosis and prognosis of PCa. PanHW et al. found in the IHC samples of human normal prostate tissues, PCa tissues and PCa cells, that TPX2 was not expressed in normal tissues but highly expressed in PCa, which was consistent with our findings.(50). TPX2 can be used as prognosis biomarkers as well. It shows that the time to biochemical recurrence-free was shorter in the TPX2 high expression group, which indicating a poor prognosis(51).

\subsubsection{The relatively less-studied PCa biomarkers}

Though lack of validation by HPA database, the identified relatively less-studied PCa biomarkers from our bioinformatics study would provide novel and valuable insights in the diagnosis and prognosis of PCa.

Baculoviral IAP repeat-containing protein 5(BIRC5) is multitasking protein with dual roles in promoting cell proliferation and preventing apoptosis(52). Its role in the carcinogenesis and prognosis of PCa is not quite clear. According to Adisetiyo et al.'s finding, the expression of BIRC5 is directly proportional to the tumor volume in a mouse model of PCa (53). According to a latest large clinical study, BIRC5 was found increased in PCa especially advanced cases but still not clear whether it is an independent PCa biomarker(54). These results indicate consistence with our findings, that BIRC5 is highly expressed in PCa and is a high-risk gene for the prognosis of PCa.

Ribonucleotide reductase regulatory subunit M2 (RRM2) is an enzyme that regulate DNA synthesis and repair, which is important in carcinogenesis(55). RRM2 was highly expressed in patients with poorly differentiated PCa or advanced PCa $(p<0.05)$, and RRM2 would be a prognostic marker for the risk of recurrence in patients with low-risk $\mathrm{PCa}(56)$. In patients with Gleason score 4-7 and no invasion of the tumor into the prostate capsule, Cox proportional hazards analysis revealed that the risk of recurrence was positively correlated with RRM2 protein expression levels(56). In a recent preclinical and clinical study, RRM2 was found to be a driven factor for poor PCa prognosis outcomes and knockdown RRM2 could prohibit the PCa development(57). Although RRM2 was not found in either PCa or normal prostate IHC samples in HPA database, RRM2 is high likely to be a vital diagnosis and prognosis biomarker of PCa based on our analysis and recent findings.

DNA topoisomerase 2-alpha(TOP2A) encodes topoisomerase lla, which controls DNA topology as well as cell cycle progression(58). According to Labbé et al., TOP2A can be a marker for PCa metastasis, and they found that TOP2A levels were highly expressed in patients with metastatic PCa by cohort studies, in $\mathrm{IHC}$ staining of tissue sections from patients with different stages of PCa, and in mice PCa cells (59). 
According to Resende et.al., TOP2A can exist as a prognostic marker in PCa (60). In PCa patients with high Gleason scores (i.e., poorly differentiated PCa), the levels of TOP2A were significantly higher. And biochemical recurrence-free survival was significantly shorter in patients with high TOP2A expression ( $P$ $=0.001)(60)$. Our results consistently found TOP2A was highly expressed in PCa patients, and was a high-risk gene for PCa prognosis.

Ubiquitin-conjugating enzyme E2 C(UBE2C) is an anaphase-promoting complex/cyclosome (APC/C) specific ubiquitin-conjugating enzyme(61). Currently, there are few studies on the mechanism of UBE2C in $\mathrm{PCa}$. It has been confirmed that UBE2C levels regulated by miR-381-3p are positively correlated with the proliferation of PCa cells(62). UBE2C exists as a target gene for Androgen receptor (AR), and in castration resistant $\mathrm{PCa}(\mathrm{CRPC})$, the expression of UBE2C positively correlates with the level of Androgen receptor splice variant 7 and promotes the progression of $\operatorname{CRPC}(63)$. According to the study form ours and the peers, UBE2C would probably be a promising biomarker for the diagnosis and prognosis of PCa.

ZW10 interactor(ZWINT) refers to a protein involved in kinetochore function(64). There are less clinical proves to illustrate the mechanistic link between ZWINT and PCa. In a bioinformatics analysis, ZWINT was found to be increased in PCa and was negatively correlated with miR-1(65). In a study of PCa microarray data, ZWINT was also found to be upregulated in PCa and correlated with the PCa grade(66). In our study, ZWINT is highly expressed in PCa and is positively correlated with poor prognosis, which can be used as a potential biomarker for prostate cancer diagnosis and prognosis. However more follow-up experiments are needed to verify and discover its role in PCa.

\subsection{Targets of natural products for treatment of PCa}

Our study identified four potential curcumin targets (EP300, EGFR, RELA, NFKB1), 7 paclitaxel targets (PTEN, EGFR, ERBB2, TP53, KRAS, AR, AKT1), and 2 ursolic acid targets (GSK3B, RELA) that could be used for $\mathrm{PCa}$ treatment. Peer research results suggest they may be potential PCa-natural products targets.

\subsubsection{Targets of curcumin for treatment of PCa}

E1A-associated protein P300(EP300) plays an important role in cell proliferation, cell cycle regulation, apoptosis, and DNA damage repair(67). It is highly expressed in advanced PCa such as CRPC and is accepted as targets for PCa treatment $(68,69)$. Though there is no direct evidence, EP300 could probably be a potential PCa target for curcumin's inhibition. It is found that Curcumin could prohibit the acetyltransferase function of EP300 in Human osteosarcoma cells(70).By a silico docking analysis, curcumin analogue was found to molecularly target EP300 and inhibit its acetyltransferase ability(71).

Epidermal growth factor receptor (EGFR) is actively involved in the carcinogenesis processes such as tumor cell proliferation, angiogenesis, tumor invasion, metastasis and apoptosis(72). Curcumin may exert its anti-PCa effects through targeting EGFR. Co-delivery of curcumin and docetaxel by EGFR ligand nanoparticles have shown therapeutic effects on $\mathrm{PCa}(73)$. Synergetically administration of curcumin and 
phenylethyl isothiocyanate were found to inhibit the growth of PCa PC-3 cells most probably through targeting EGFR, Akt and NF-kappaB pathways(74).

Transcription factor p65(RELA) is an isoform of the nuclear factor kappa-B protein, also known as p65, which can regulate inflammation, cell differentiation, proliferation and apoptosis(75). Only one reference was found indicating curcumin could inhibit PCa through RELA. In analyzing human PCa cell lines (PC3, DU145, LNCap), it was found that curcumin could inhibit the growth of androgen-independent PCa cells through p65 inhibition (76). Our network pharmacology analysis highly suggests the value of studying RELA as a novel potential PCa-curcumin target.

Nuclear factor NF-kappa-B p105 subunit(NFKB1) is another isoform of the kappa-B protein, also called p105/p50, which plays an important role in modulating innate and adaptive immunity, cell proliferation and apoptosis(77). Activation and translocation of NFKB1 is correlated with the progression of $\mathrm{PCa}(78)$. In human PCa cells, curcumin was found to inhibit the growth of LNCaP and PC3 cells by blocking the transactivation of NFKB1 $(78,79)$.

\subsubsection{Targets of paclitaxel for treatment of $\mathrm{PCa}$}

Phosphatase and tensin homolog deleted on chromosome ten(PTEN) plays an important role in carcinogenesis and tumor metastasis suppression(80). Combination application of Naringin and paclitaxel were found to inhibit growth of DU145 PCa cells by increasing PTEN and decreasing NFKB1(81). Paclitaxel could induce more apoptosis in PTEN-positive 22Rv1 PCa cells than these cells with PTEN knockdown (82). These results probably demonstrate that PTEN can be used as a target for paclitaxel treatment of $\mathrm{PCa}$.

EGFR is a common potential target for both curcumin and paclitaxel in the treatment of PCa. Coadministration of EGFR inhibitor and paclitaxel indicated significant inhibition in the tumor growth and metastasis in human PCa transplanted nude mice model(83). Upregulated EGFR and actin level were found to be the causes of paclitaxel resistance to PCa cells(84).

Erb-b2 receptor tyrosine kinase 2 (ERBB2 or HER2) is a member of the EGFR family, which is overexpressed in $\mathrm{PCa}$ and bone metastasis of $\mathrm{PCa}(85)$. Co-administration of pacilitaxel and anti-HER2 emulsion $(10 \mathrm{mg} / \mathrm{kg}$ ) every week for 3 weeks significantly inhibited the PCa growth in PCa transplanted nude mice model(86).

Tumor protein 53 (TP53) is a tumor suppressor gene which is mainly involved in regulation of apoptosis and cell cycles (87). In an interactome and topology comparison experiments in PC-3 PCa cells, TP53 was identified as one of the vital pathways for paclitaxel in anti-PCa(88).

Kirsten rat sarcoma viral oncogene homolog (KRAS) is one of the three members of the RAS family(KRAS, HRAS and NRAS) with the highest probability of mutation in human tumors among them(89). Mutations of KRAS are associated with a variety of malignant tumors, including lung cancer, pancreatic cancer, and colorectal cancer(90). It is still unknown whether paclitaxel exert its anti-PCa 
effects via KRAS. However, though not commonly mutated in PCa, KRAS was believed to play an important role in bone metastasis of $\mathrm{PCa}(91)$. It is worthwhile to do further study to verify the hypothesis.

Androgen receptor (AR) is highly expressed in primary and metastatic $\mathrm{PCa}$, which can regulate the proliferation, apoptosis, and metastasis of $\mathrm{PCa}(92)$. Jiang et al. found that paclitaxel was able to target and inhibit the transcriptional activity of AR in the 22Rv1 PCa cell line, a CRPC cell model(93). In another CRPC cell line 22RV1, paclitaxel was found to inhibit AR transcription by inducing nuclear accumulation of FOXO1(94).

AKT serine/threonine kinase 1 (AKT1), a family member of AKT (protein kinase B) and core component of the PI3K/AKT signaling pathway, plays an important role in PCa carcinogenesis(95). Co-administration of AKT inhibitors and paclitaxel are very powerful in treating triple negative breast cancer with mutated $\operatorname{AKT1}(96,97)$. However, the correlation between AKT1, paclitaxel and PCa is still unknown. Our findings may provide novel direction in the future study.

\subsubsection{Targets of ursolic acid for treatment of PCa}

Glycogen synthase kinase $3 \beta$ (GSK3B) is involved in biological processes such as energy metabolism, inflammation, and apoptosis(98). Only 1 report so far indicates the correlation between urosolic acid and PCa and GSK3B. Ursolic acid was found to induce apoptosis to PC-3, LNCaP and DU145 PCa cells with enhanced phosphorylation GSK3B and the apoptosis effects can be reversed by GSK3B inhibitor SB216763(99). In HepG2 liver cancer cells, ursolic acid can also induce apoptosis by targeting and regulating the phosphorylation of GSK3B(100). Hence GSK3B is a probably a novel potential target for ursolic acid in the treatment of prostate cancer.

Transcription factor p65(RELA) is a common potential PCa therapeutic target of curcumin and ursolic acid, which deserves further studies in the mechanism of action. The direct proof of ursolic acid inhibit PCa through RELA is limited. Shanmugam et al. found ursolic acid could induce apoptosis in DU145, LNCaP PCa cells in a dose-dependent manner through inhibiting the activity of NF-kB and phosphorylation of p65(RELA)(101).

\section{Conclusions}

In this study, by analyzing two PCa datasets from the GEO database and validated by GEPIA and HPA, 9 genes were identified to be closely associated with the development and prognosis of $\mathrm{PCa}$. All the nine markers indicated poor prognosis for PCa patients verified by GEPIA. The 4 identified biomarkers verified by HPA (CENPF, TPX2, TK1 and CCNB1) indicate the rationality of our study design and the identified 5 relatively less-studied PCa biomarkers (RRM2, UBE2C, TOP2A, BIRC5 and ZWINT) would provide novel and valuable insights in the in the diagnosis and prognosis of $\mathrm{PCa}$. The carcinogenesis of PCa is found to be mainly associated with hepatic fibrosis pathway, ILK signaling, NRF2-mediated oxidative stress response and many others. By network pharmacology, after the integration of data on small molecule natural products acting on PCa, 4 targets of curcumin (EP300, RELA, EGFR, NFKB1), 7 targets of 
paclitaxel (PTEN, EGFR, ERBB2, TP53, KRAS, AR, AKT1), and 2 targets of ursolic acid (GSK3B, RELA) were identified.

Our study reveals potential key biomarkers for the diagnosis and prognosis of PCa and demonstrates three valuable natural products for molecular targeting of $\mathrm{PCa}$, which laid a promising foundation for further study of novel biomarkers and molecularly targeted drugs for PCa.

\section{Abbreviations}




\begin{tabular}{|c|c|}
\hline Abbreviations & Full name \\
\hline Aclbi & Assistant for Clinical Bioinformatics \\
\hline AR & Androgen receptor \\
\hline $\mathrm{BC}$ & Betweenness Centrality \\
\hline BPs & Biological processes \\
\hline CCs & Cellular components \\
\hline CLC & Closeness Centrality \\
\hline CRPC & Castration Resistant Prostate Cancer \\
\hline DAVID & The Database for Annotation, Visualization and Integrated Discovery \\
\hline DC & Degree Centrality \\
\hline DEGs & differentially expressed genes \\
\hline EC & Eigenvector Centrality \\
\hline GEO & Gene Expression Omnibus \\
\hline GEPIA & Gene Expression Profiling Interactive Analysis \\
\hline GO & Gene Ontology \\
\hline GTEx & The Genotype-Twassue Expression \\
\hline HPA & The Human Protein Atlas \\
\hline $\mathrm{IHC}$ & Immunohistochemical \\
\hline IPA & QIAGEN Ingenuity Pathway Analysis \\
\hline KEGG & Kyoto Encyclopedia of Genes and Genomes \\
\hline LAC & Local average connectivity-based method \\
\hline MFs & Molecular functions \\
\hline NC & Network Centrality \\
\hline OMIM & Online Mendelian Inheritance in Man \\
\hline $\mathrm{PCa}$ & Prostate Cancer \\
\hline PDB & Protein Data Bank \\
\hline $\mathrm{PI} 3 \mathrm{~K}$ & Phosphatidylinositol 3 kinase \\
\hline PPI & Protein-Protein Interaction \\
\hline PRAD & Prostate Adenocarcinoma \\
\hline
\end{tabular}




\begin{tabular}{|ll|}
\hline Abbreviations & Full name \\
\hline PSA & prostate-specific antigen \\
\hline TCGA & The Cancer Genome Atlas \\
\hline TTD & Therapeutic Target Database \\
\hline
\end{tabular}

\section{Declarations}

\section{Ethics statement}

Our research was approved by the Ethics Committee of Medical College at Yangzhou University, and followed the principles of the Declaration of Helsinki. All datasets analyzed were obtained from publicly available databases, hence written informed consent was not applicable.

\section{Consent for publication}

Not applicable

\section{Availability of data and materials}

All data involved in the study are included in this manuscript and its supplementary files.

\section{Competing Interests}

The authors declare that there are no conflicts of interest.

\section{Author Contribution}

WL designed the project, analyzed the data, drafted and revised the manuscript. WX did most of the bioinformatics analysis and modified the manuscript. KS, FW and TWW performed the literature search and revised the manuscript.

\section{Funding}

This work was supported by the National Natural Science Foundation of China (81973518), Talents startup fund from Yangzhou University (137011474), and Postgraduate Research \& Practice Innovation Program of Jiangsu Province (18252757292).

\section{Acknowledgments}

We thank the Hiplot team and the Aclbi team for providing technical assistance and valuable tools for data Analysis and visualization. The authors express sincere gratitude to all members of Dr. Wenji Li's laboratory for their helpful discussions. 


\section{References}

1. Bray F, Ferlay J, Soerjomataram I, Siegel RL, Torre LA, Jemal A. Global cancer statistics 2018: GLOBOCAN estimates of incidence and mortality worldwide for 36 cancers in 185 countries. Cancer J Clin. 2018;68(6):394-424.

2. Liu X, Yu C, Bi Y, Zhang ZJ. Trends and age-period-cohort effect on incidence and mortality of prostate cancer from 1990 to 2017 in China. Public Health. 2019;172:70-80.

3. Lakes J, Arsov C. [PSA screening and molecular markers]. Urologe A. 2019;58(5):486-93.

4. Giri VN, Knudsen KE, Kelly WK, Abida W, Andriole GL, Bangma CH, et al. Role of Genetic Testing for Inherited Prostate Cancer Risk: Philadelphia Prostate Cancer Consensus Conference 2017. J Clin Oncol. 2018;36(4):414 - 24.

5. Oncology CA-CAGCCJC. Chinese expert consensus on genomic testing of prostate cancer patients (the 2020 edition). 2020;30(7):551-60.

6. Wallis CJD, Saskin R, Choo R, Herschorn S, Kodama RT, Satkunasivam R, et al. Surgery Versus Radiotherapy for Clinically-localized Prostate Cancer: A Systematic Review and Meta-analysis. Eur Urol. 2016;70(1):21-30.

7. Teo MY, Rathkopf DE, Kantoff P. Treatment of Advanced Prostate Cancer. Annu Rev Med. 2019;70:479-99.

8. Mansinho A, Macedo D, Fernandes I, Costa L. Castration-Resistant Prostate Cancer: Mechanisms, Targets and Treatment. Adv Exp Med Biol. 2018;1096:117-33.

9. Lee YT, Tan YJ, Oon CE. Molecular targeted therapy: Treating cancer with specificity. Eur J Pharmacol. 2018;834:188-96.

10. Grüllich $C$, Nößner E, Pfister D, Grünwald V. [Targeted molecular therapy and immunotherapy for prostate cancer]. Urologe A. 2020;59(6):687-94.

11. Rodrigues T, Reker $D$, Schneider P, Schneider G. Counting on natural products for drug design. Nat Chem. 2016;8(6):531-41.

12. Kotha RR, Luthria DL. Curcumin. Biological, Pharmaceutical, Nutraceutical, and Analytical Aspects. Molecules. 2019;24(16).

13. Yang C-PH, Horwitz SB. Taxol: The First Microtubule Stabilizing Agent. Int J Mol Sci. 2017;18(8).

14. Weaver BA. How Taxol/paclitaxel kills cancer cells. Mol Biol Cell. 2014;25(18):2677-81.

15. Li W, Sun K, Hu F, Chen L, Zhang X, Wang F, et al. Protective effects of natural compounds against oxidative stress in ischemic diseases and cancers via activating the Nrf2 signaling pathway: A mini review. J Biochem Mol Toxicol. 2021;35(3):e22658.

16. Varambally S, Yu J, Laxman B, Rhodes DR, Mehra R, Tomlins SA, et al. Integrative genomic and proteomic analysis of prostate cancer reveals signatures of metastatic progression. Cancer Cell. 2005;8(5):393-406. 
17. Mortensen MM, Høyer S, Lynnerup A-S, Ørntoft TF, Sørensen KD, Borre M, et al. Expression profiling of prostate cancer tissue delineates genes associated with recurrence after prostatectomy. Sci Rep. 2015;5:16018.

18. Dennis G, Sherman BT, Hosack DA, Yang J, Gao W, Lane HC, et al. DAVID: Database for Annotation, Visualization, and Integrated Discovery. Genome Biol. 2003;4(5):P3.

19. Chen L, Zhang Y-H, Wang S, Zhang Y, Huang T, Cai Y-D. Prediction and analysis of essential genes using the enrichments of gene ontology and KEGG pathways. PLoS One. 2017;12(9):e0184129.

20. von Mering C, Huynen M, Jaeggi D, Schmidt S, Bork P, Snel B. STRING: a database of predicted functional associations between proteins. Nucleic Acids Res. 2003;31(1):258-61.

21. Chin $\mathrm{C}-\mathrm{H}$, Chen $\mathrm{S}-\mathrm{H}, \mathrm{Wu} \mathrm{H}-\mathrm{H}, \mathrm{Ho} \mathrm{C}-\mathrm{W}, \mathrm{Ko}$ M-T, Lin C-Y. cytoHubba: identifying hub objects and subnetworks from complex interactome. BMC Syst Biol. 2014;8(Suppl 4):11.

22. Su G, Morris JH, Demchak B, Bader GD. Biological network exploration with Cytoscape 3. Curr Protoc Bioinformatics. 2014;47:8.13.1-8.1324..

23. Bader GD, Hogue CWV. An automated method for finding molecular complexes in large protein interaction networks. BMC Bioinformatics. 2003;4:2.

24. Tang Z, Li C, Kang B, Gao G, Li C, Zhang Z. GEPIA: a web server for cancer and normal gene expression profiling and interactive analyses. Nucleic Acids Res. 2017;45:W1.

25. Uhlen M, Zhang C, Lee S, Sjöstedt E, Fagerberg L, Bidkhori G, et al. A pathology atlas of the human cancer transcriptome. Science. 2017;357:6352.

26. Li T, Fu J, Zeng Z, Cohen D, Li J, Chen Q, et al. TIMER2.0 for analysis of tumor-infiltrating immune cells. Nucleic Acids Res. 2020;48(W1):W509-W14.

27. Stelzer G, Rosen N, Plaschkes I, Zimmerman S, Twik M, Fishilevich S, et al. The GeneCards Suite: From Gene Data Mining to Disease Genome Sequence Analyses. Curr Protoc Bioinformatics. 2016;54.

28. Amberger JS, Hamosh A. Searching Online Mendelian Inheritance in Man (OMIM): A Knowledgebase of Human Genes and Genetic Phenotypes. Curr Protoc Bioinformatics. 2017;58.

29. Barbarino JM, Whirl-Carrillo M, Altman RB, Klein TE. PharmGKB: A worldwide resource for pharmacogenomic information. Wiley Interdiscip Rev Syst Biol Med. 2018;10(4):e1417.

30. Wishart DS, Feunang YD, Guo AC, Lo EJ, Marcu A, Grant JR, et al. DrugBank 5.0: a major update to the DrugBank database for 2018. Nucleic Acids Res. 2018;46(D1):D1074-D82.

31. Chen X, Ji ZL, Chen YZ. TTD: Therapeutic Target Database. Nucleic Acids Res. 2002;30(1):412-5.

32. Gilson MK, Liu T, Baitaluk M, Nicola G, Hwang L, Chong J. BindingDB in 2015: A public database for medicinal chemistry, computational chemistry and systems pharmacology. Nucleic Acids Res. 2016;44(D1):D1045-D53.

33. Kim S. Getting the most out of PubChem for virtual screening. Expert Opin Drug Discov. 2016;11(9):843-55. 
34. Gaulton A, Bellis LJ, Bento AP, Chambers J, Davies M, Hersey A, et al. ChEMBL: a large-scale bioactivity database for drug discovery. Nucleic Acids Res. 2012;40(Database issue):D1100-D7.

35. Divisi D, Di Leonardo G, Zaccagna G, Crisci R. Basic statistics with Microsoft Excel: a review. J Thorac Dis. 2017;9(6):1734-40.

36. Tang Y, Li M, Wang J, Pan Y, Wu F-X. CytoNCA: a cytoscape plugin for centrality analysis and evaluation of protein interaction networks. Biosystems. 2015;127:67-72.

37. Burley SK, Berman HM, Kleywegt GJ, Markley JL, Nakamura H, Velankar S. Protein Data Bank (PDB): The Single Global Macromolecular Structure Archive. Methods Mol Biol. 2017;1607:627-41.

38. Seeliger D, de Groot BL. Ligand docking and binding site analysis with PyMOL and Autodock/Vina. J Comput Aided Mol Des. 2010;24(5):417-22.

39. Morris GM, Huey R, Lindstrom W, Sanner MF, Belew RK, Goodsell DS, et al. AutoDock4 and AutoDockTools4: Automated docking with selective receptor flexibility. J Comput Chem. 2009;30(16):2785-91.

40. Chandrashekar DS, Bashel B, Balasubramanya SAH, Creighton CJ, Ponce-Rodriguez I, Chakravarthi BVSK, et al. UALCAN: A Portal for Facilitating Tumor Subgroup Gene Expression and Survival Analyses. Neoplasia. 2017;19(8):649-58.

41. Chen Q, Hu J, Deng J, Fu B, Guo J. Bioinformatics Analysis Identified Key Molecular Changes in Bladder Cancer Development and Recurrence. Biomed Res Int. 2019;2019:3917982.

42. Niranjan KC, Tayaar A, Kumar GS, Krishnapillai R, Hallikeri K, Hunasgi S. Immunohistochemical Expression of Cyclin B1 in Epithelial Hyperplasia, Dysplasia and Oral Squamous Cell Carcinomas - A Comparative Study. J Clin Diagn Res. 2016;10(9):ZC85-90.

43. Gomez LA, de Las Pozas A, Reiner T, Burnstein K, Perez-Stable C. Increased expression of cyclin B1 sensitizes prostate cancer cells to apoptosis induced by chemotherapy. Mol Cancer Ther. 2007;6(5):1534-43.

44. Shahid M, Lee MY, Piplani H, Andres AM, Zhou B, Yeon A, et al. Centromere protein F (CENPF), a microtubule binding protein, modulates cancer metabolism by regulating pyruvate kinase $\mathrm{M} 2$ phosphorylation signaling. Cell Cycle. 2018;17(24):2802-18.

45. Göbel C, Özden C, Schroeder C, Hube-Magg C, Kluth M, Möller-Koop C, et al. Upregulation of centromere protein $\mathrm{F}$ is linked to aggressive prostate cancers. Cancer Manag Res. 2018;10:5491504.

46. Bitter EE, Townsend MH, Erickson R, Allen C, O'Neill KL. Thymidine kinase 1 through the ages: a comprehensive review. Cell Biosci. 2020;10(1):138.

47. Jagarlamudi KK, Shaw M. Thymidine kinase 1 as a tumor biomarker: technical advances offer new potential to an old biomarker. Biomark Med. 2018;12(9):1035-48.

48. Jagarlamudi KK, Hansson LO, Eriksson S. Breast and prostate cancer patients differ significantly in their serum Thymidine kinase 1 (TK1) specific activities compared with those hematological malignancies and blood donors: implications of using serum TK1 as a biomarker. BMC Cancer. 2015;15:66. 
49. Wang M, Yang M, Deng B. Weakens the Tumorigenesis of Colon Cancer Through Targeting. Cancer Biother Radiopharm. 2020.

50. Pan H-W, Su H-H, Hsu C-W, Huang G-J, Wu TT-L. Targeted TPX2 increases chromosome missegregation and suppresses tumor cell growth in human prostate cancer. Onco Targets Ther. 2017;10:3531-43.

51. Zou J, Huang R-Y, Jiang F-N, Chen D-X, Wang C, Han Z-D, et al. Overexpression of TPX2 is associated with progression and prognosis of prostate cancer. Oncol Lett. 2018;16(3):2823-32.

52. Hmeljak J, Erčulj N, Dolžan V, Kern I, Cör A. BIRC5 promoter SNPs do not affect nuclear survivin expression and survival of malignant pleural mesothelioma patients. J Cancer Res Clin Oncol. 2011;137(11):1641-51.

53. Adisetiyo H, Liang M, Liao C-P, Aycock-Williams A, Cohen MB, Xu S, et al. Loss of survivin in the prostate epithelium impedes carcinogenesis in a mouse model of prostate adenocarcinoma. PLoS One. 2013;8(7):e69484.

54. Hennigs JK, Minner S, Tennstedt P, Löser R, Huland H, Klose H, et al. Subcellular Compartmentalization of Survivin is Associated with Biological Aggressiveness and Prognosis in Prostate Cancer. Sci Rep. 2020;10(1):3250.

55. Li J, Pang J, Liu Y, Zhang J, Zhang C, Shen G, et al. Suppression of RRM2 inhibits cell proliferation, causes cell cycle arrest and promotes the apoptosis of human neuroblastoma cells and in human neuroblastoma RRM2 is suppressed following chemotherapy. Oncol Rep. 2018;40(1):355-60.

56. Huang Y, Liu X, Wang Y-H, Yeh S-D, Chen C-L, Nelson RA, et al. The prognostic value of ribonucleotide reductase small subunit $\mathrm{M} 2$ in predicting recurrence for prostate cancers. Urol Oncol. 2014;32(1):51.e9-.19.

57. Mazzu YZ, Armenia J, Chakraborty G, Yoshikawa Y, Coggins SA, Nandakumar S, et al. A Novel Mechanism Driving Poor-Prognosis Prostate Cancer: Overexpression of the DNA Repair Gene, Ribonucleotide Reductase Small Subunit M2 (RRM2). Clin Cancer Res. 2019;25(14):4480-92.

58. Lee JH, Berger JM. Cell Cycle-Dependent Control and Roles of DNA Topoisomerase II. Genes (Basel). 2019;10(11).

59. Labbé DP, Sweeney CJ, Brown M, Galbo P, Rosario S, Wadosky KM, et al. TOP2A and EZH2 Provide Early Detection of an Aggressive Prostate Cancer Subgroup. Clin Cancer Res. 2017;23(22):7072-83.

60. de Resende MF, Vieira S, Chinen LTD, Chiappelli F, da Fonseca FP, Guimarães GC, et al. Prognostication of prostate cancer based on TOP2A protein and gene assessment: TOP2A in prostate cancer. J Transl Med. 2013;11:36.

61. Wang Y, Wang J, Tang Q, Ren G. Identification of UBE2C as hub gene in driving prostate cancer by integrated bioinformatics analysis. PLoS One. 2021;16(2):e0247827.

62. Hu J, Wu X, Yang C, Rashid K, Ma C, Hu M, et al. Anticancer effect of icaritin on prostate cancer via regulating miR-381-3p and its target gene UBE2C. Cancer Med. 2019;8(18):7833-45.

63. Hu R, Lu C, Mostaghel EA, Yegnasubramanian S, Gurel M, Tannahill C, et al. Distinct transcriptional programs mediated by the ligand-dependent full-length androgen receptor and its splice variants in 
castration-resistant prostate cancer. Cancer Res. 2012;72(14):3457-62.

64. Miller WR. Clinical, pathological, proliferative and molecular responses associated with neoadjuvant aromatase inhibitor treatment in breast cancer. J Steroid Biochem Mol Biol. 2010;118(4-5):273-6.

65. Xie ZC, Huang JC, Zhang LJ, Gan BL, Wen DY, Chen G, et al. Exploration of the diagnostic value and molecular mechanism of miR-1 in prostate cancer: A study based on meta-analyses and bioinformatics. Mol Med Rep. 2018;18(6):5630-46.

66. Song ZY, Chao F, Zhuo Z, Ma Z, Li W, Chen G. Identification of hub genes in prostate cancer using robust rank aggregation and weighted gene co-expression network analysis. Aging. 2019;11(13):4736-56.

67. Ring A, Kaur P, Lang JE. EP300 knockdown reduces cancer stem cell phenotype, tumor growth and metastasis in triple negative breast cancer. BMC Cancer. 2020;20(1):1076.

68. Xiang Q, Wang C, Zhang Y, Xue X, Song M, Zhang C, et al. Discovery and optimization of 1-(1H-indol1-yl)ethanone derivatives as CBP/EP300 bromodomain inhibitors for the treatment of castrationresistant prostate cancer. Eur J Med Chem. 2018;147:238-52.

69. Zou LJ, Xiang QP, Xue XQ, Zhang C, Li CC, Wang C, et al. Y08197 is a novel and selective CBP/EP300 bromodomain inhibitor for the treatment of prostate cancer. Acta pharmacologica Sinica. 2019;40(11):1436-47.

70. Pietrocola F, Lachkar S, Enot DP, Niso-Santano M, Bravo-San Pedro JM, Sica V, et al. Spermidine induces autophagy by inhibiting the acetyltransferase EP300. Cell Death Differ. 2015;22(3):509-16.

71. Kandagalla S, Shekarappa SB, Rimac H, Grishina MA, Potemkin VA, Hanumanthappa M. Computational insights into the binding mode of curcumin analogues against EP300 HAT domain as potent acetyltransferase inhibitors. J Mol Graph Model. 2020;101:107756.

72. Wang Z. ErbB Receptors and Cancer. Methods Mol Biol. 2017;1652.

73. Yan J, Wang Y, Jia Y, Liu S, Tian C, Pan W, et al. Co-delivery of docetaxel and curcumin prodrug via dual-targeted nanoparticles with synergistic antitumor activity against prostate cancer. Biomedicine pharmacotherapy = Biomedecine pharmacotherapie. 2017;88:374-83.

74. Kim JH, Xu C, Keum YS, Reddy B, Conney A, Kong AN. Inhibition of EGFR signaling in human prostate cancer PC-3 cells by combination treatment with beta-phenylethyl isothiocyanate and curcumin. Carcinogenesis. 2006;27(3):475-82.

75. DiDonato JA, Mercurio F, Karin M. NF-kB and the link between inflammation and cancer. Immunol Rev. 2012;246(1):379-400.

76. Li J, Xiang S, Zhang Q, Wu J, Tang Q, Zhou J, et al. Combination of curcumin and bicalutamide enhanced the growth inhibition of androgen-independent prostate cancer cells through SAPK/JNK and MEK/ERK1/2-mediated targeting NF-KB/p65 and MUC1-C. J Exp Clin Cancer Res. 2015;34:46.

77. Perkins ND. The diverse and complex roles of NF-kappaB subunits in cancer. Nat Rev Cancer. 2012;12(2):121-32. 
78. Fernández-Martínez AB, Carmena MJ, Bajo AM, Vacas E, Sánchez-Chapado M, Prieto JC. VIP induces NF-KB1-nuclear localisation through different signalling pathways in human tumour and non-tumour prostate cells. Cellular signalling. 2015;27(2):236-44.

79. Fernández-Martínez AB, Collado B, Bajo AM, Sánchez-Chapado M, Prieto JC, Carmena MJ. Vasoactive intestinal peptide induces cyclooxygenase-2 expression through nuclear factor-kappaB in human prostate cell lines Differential time-dependent responses in cancer progression. Molecular cellular endocrinology. 2007;270(1-2):8-16.

80. Georgescu MM. PTEN Tumor Suppressor Network in PI3K-Akt Pathway Control. Genes Cancer. 2010;1(12):1170-7.

81. Erdogan S, Doganlar O, Doganlar ZB, Turkekul K. Naringin sensitizes human prostate cancer cells to paclitaxel therapy. Prostate international. 2018;6(4):126-35.

82. Gan Y, Chen Q, Lei Y. Regulation of paclitaxel sensitivity in prostate cancer cells by PTEN/maspin signaling. Oncol Lett. 2017;14(4):4977-82.

83. Yazici S, Kim SJ, Busby JE, He J, Thaker P, Yokoi K, et al. Dual inhibition of the epidermal growth factor and vascular endothelial growth factor phosphorylation for antivascular therapy of human prostate cancer in the prostate of nude mice. Prostate. 2005;65(3):203-15.

84. Li Y, Mizokami A, Izumi K, Narimoto K, Shima T, Zhang J, et al. CTEN/tensin 4 expression induces sensitivity to paclitaxel in prostate cancer. Prostate. 2010;70(1):48-60.

85. Day KC, Lorenzatti Hiles G, Kozminsky M, Dawsey SJ, Paul A, Broses LJ, et al. HER2 and EGFR Overexpression Support Metastatic Progression of Prostate Cancer to Bone. Cancer Res. 2017;77(1):74-85.

86. Goldstein D, Gofrit O, Nyska A, Benita S. Anti-HER2 cationic immunoemulsion as a potential targeted drug delivery system for the treatment of prostate cancer. Cancer research. 2007;67(1):269-75.

87. Donehower LA, Soussi T, Korkut A, Liu Y, Schultz A, Cardenas M, et al. Integrated Analysis of TP53 Gene and Pathway Alterations in The Cancer Genome Atlas. Cell reports. 2019;28(5).

88. Delgado-Carreño C, Méndez-Callejas G. Topological properties and in vitro identification of essential nodes of the Paclitaxel and Vincristine interactomes in PC-3 cells. Biomedical journal. 2019;42(5):307-16.

89. Uprety D, Adjei AA. KRAS: From undruggable to a druggable Cancer Target. Cancer Treat Rev. 2020;89:102070.

90. Yan C, Theodorescu D. RAL GTPases: Biology and Potential as Therapeutic Targets in Cancer. Pharmacological reviews. 2018;70(1).

91. Yang Q, Lang C, Wu Z, Dai Y, He S, Guo W, et al. MAZ promotes prostate cancer bone metastasis through transcriptionally activating the KRas-dependent RaIGEFs pathway. Journal of experimental clinical cancer research: CR. 2019;38(1):391.

92. Culig Z, Santer FR. Androgen receptor signaling in prostate cancer. Cancer Metastasis Rev. 2014;33(2-3):413-27. 
93. Jiang J, Huang $\mathrm{H}$. Targeting the Androgen Receptor by Taxol in Castration-Resistant Prostate Cancer. Mol Cell Pharmacol. 2010;2(1):1-5.

94. Gan L, Chen S, Wang Y, Watahiki A, Bohrer L, Sun Z, et al. Inhibition of the androgen receptor as a novel mechanism of taxol chemotherapy in prostate cancer. Cancer Res. 2009;69(21):8386-94.

95. Karyadi DM, Zhao S, He Q, McIntosh L, Wright JL, Ostrander EA, et al. Confirmation of genetic variants associated with lethal prostate cancer in a cohort of men from hereditary prostate cancer families. International journal of cancer. 2015;136(9):2166-71.

96. Gris-Oliver A, Palafox M, Monserrat L, Brasó-Maristany F, Òdena A, Sánchez-Guixé M, et al. Genetic Alterations in the PI3K/AKT Pathway and Baseline AKT Activity Define AKT Inhibitor Sensitivity in Breast Cancer Patient-derived Xenografts. Clin Cancer Res. 2020;26(14):3720-31.

97. Schmid P, Abraham J, Chan S, Wheatley D, Brunt AM, Nemsadze G, et al. Capivasertib Plus Paclitaxel Versus Placebo Plus Paclitaxel As First-Line Therapy for Metastatic Triple-Negative Breast Cancer: The PAKT Trial. Journal of clinical oncology: official journal of the American Society of Clinical Oncology. 2020;38(5):423-33.

98. Lin J, Song T, Li C, Mao W. GSK-3 $\beta$ in DNA repair, apoptosis, and resistance of chemotherapy, radiotherapy of cancer. Biochim Biophys Acta Mol Cell Res. 2020;1867(5):118659.

99. Park JH, Kwon HY, Sohn EJ, Kim KA, Kim B, Jeong SJ, et al. Inhibition of Wnt/ $\beta$-catenin signaling mediates ursolic acid-induced apoptosis in PC-3 prostate cancer cells. Pharmacol Rep. 2013;65(5):1366-74.

100. Son H-S, Kwon HY, Sohn EJ, Lee J-H, Woo H-J, Yun M, et al. Activation of AMP-activated protein kinase and phosphorylation of glycogen synthase kinase $\beta$ mediate ursolic acid induced apoptosis in HepG2 liver cancer cells. Phytother Res. 2013;27(11):1714-22.

101. Shanmugam MK, Rajendran P, Li F, Nema T, Vali S, Abbasi T, et al. Ursolic acid inhibits multiple cell survival pathways leading to suppression of growth of prostate cancer xenograft in nude mice. $\mathrm{J}$ Mol Med (Berl). 2011;89(7):713-27.

\section{Tables}

Due to technical limitations, table 2 is only available as a download in the Supplemental Files section.

\section{Figures}




\section{a}

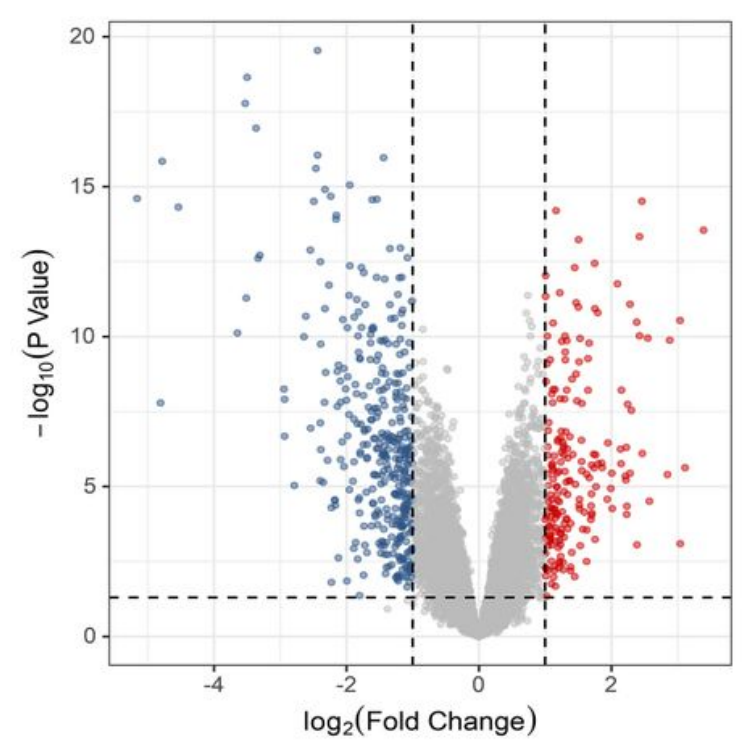

b

Group

- Down-regulated not-significant

- Up-regulated

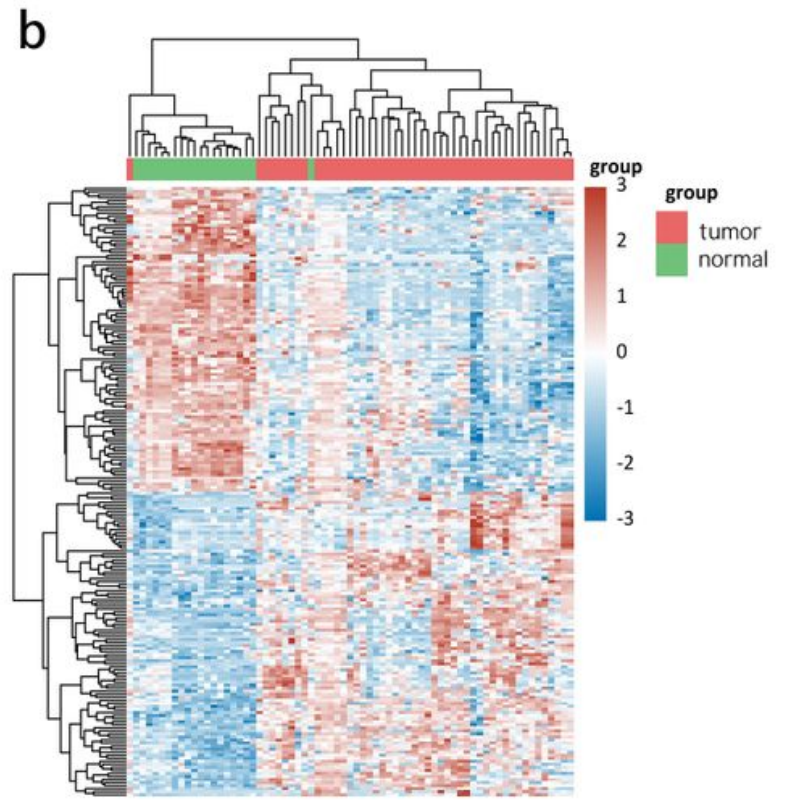

\section{Figure 1}

Identification of DEGs. (a) Volcano plots for both the GSE3325 and GSE46602 datasets. Red indicates up-regulated genes, blue indicates down-regulated genes, and grey indicates non-significant genes. (b) Heatmap for both the GSE3325 and GSE46602 datasets. Each column represents one dataset and each row represents one gene. Color changes from blue to red represents a transition from downregulation to upregulation of expression (first 100 targets displayed). 
a
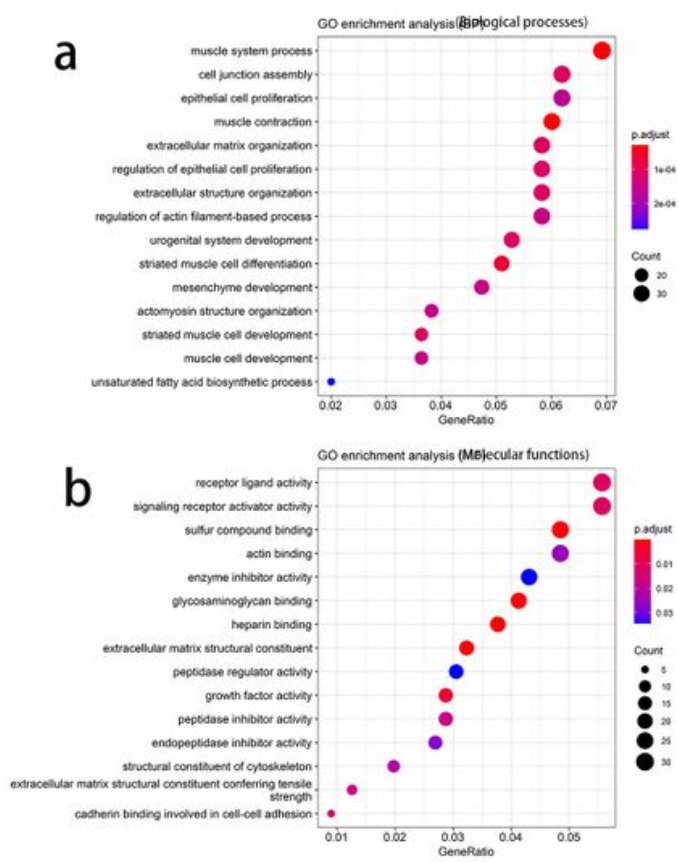

C

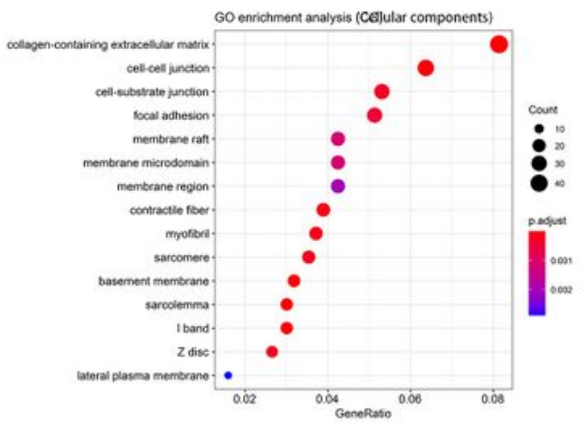

d

Wpositive $z$-score $z$-score $=0$ 政egative $z$-score $\|$ no activity pattern available $=$ Ratio

-log(p-value)

Hepatic Fibrosis / Hepatic Stellate Cell Activation

ILK Signaling

Xenobiotic Metabolism PXR Signaling Pathway

Xenobiotic Metabolism AHR Signaling Pathway

LPS/IL-1 Mediated Inhibition of RXR Function

Xenobiotic Metabolism CAR Signaling Pathway

SPINK1 Pancreatic Cancer Pathway

Aryl Hydrocarbon Receptor Signaling

NRF2-mediated Oxidative Stress Response

MSP-RON Signaling In Cancer Cells Pathway

Osteoarthritis Pathway

Agranulocyte Adhesion and Diapedesis

Nicotine Degradation II

Human Embryonic Stem Cell Pluripotency

Amyotrophic Lateral Sclerosis Signaling

MSP-RON Signaling Pathway

WNT/B-catenin Signaling

Endocannabinoid Cancer Inhibition Pathway
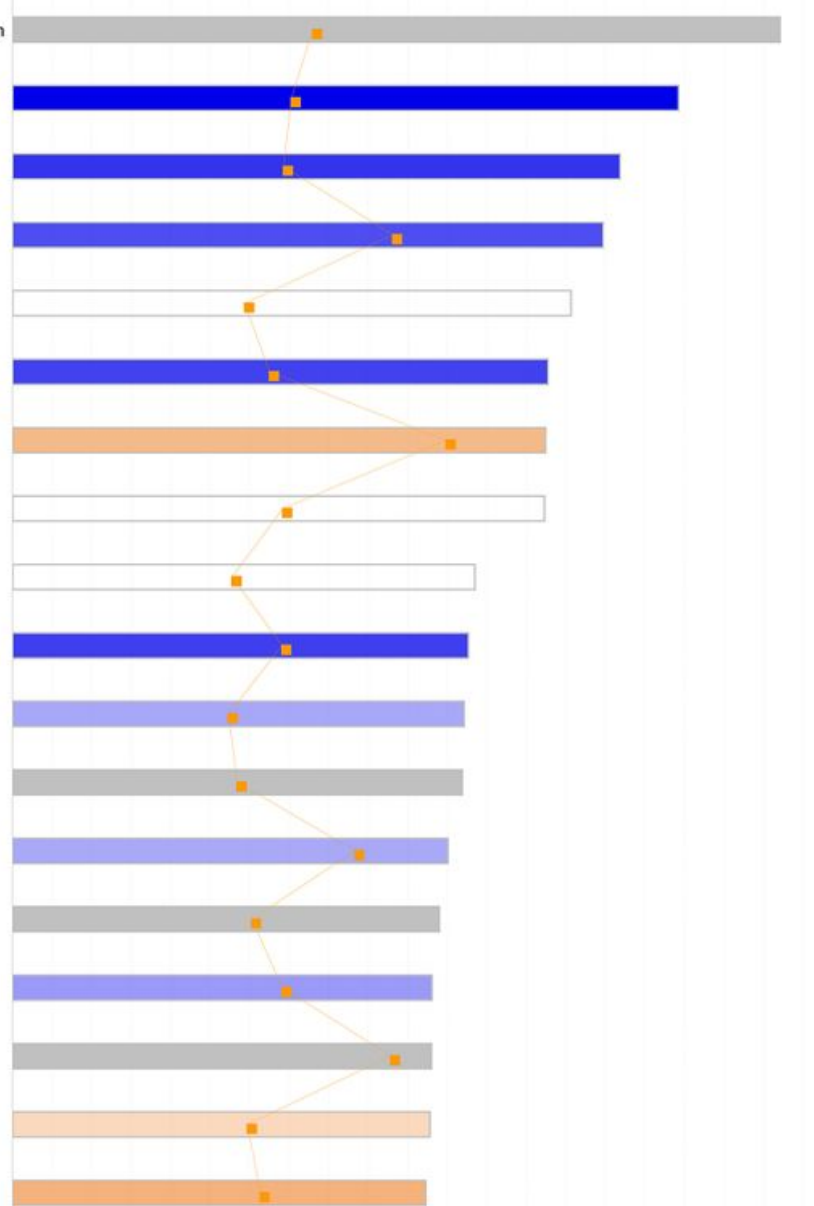

\section{Figure 2}

GO enrichment analysis and IPA pathway analysis of DEGs. (a) Biological processes(BPs) analysis of GO. (b) Molecular functions(MFs) analysis of GO . (c) Cellular components (CCs) analysis of GO. (d) IPA pathway analysis of DEGs. 

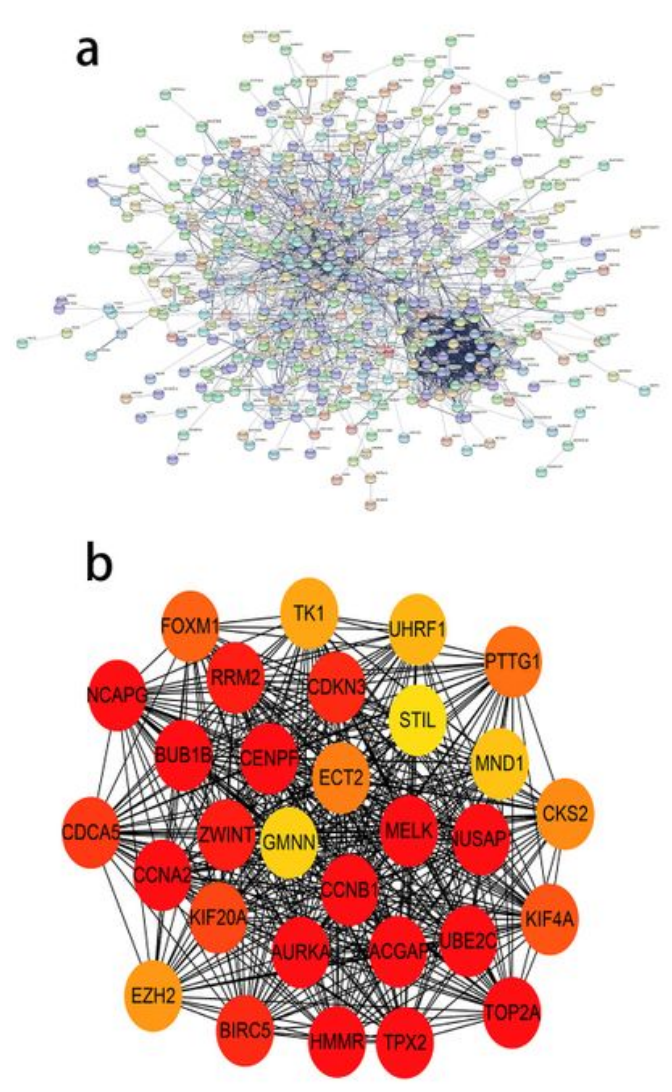
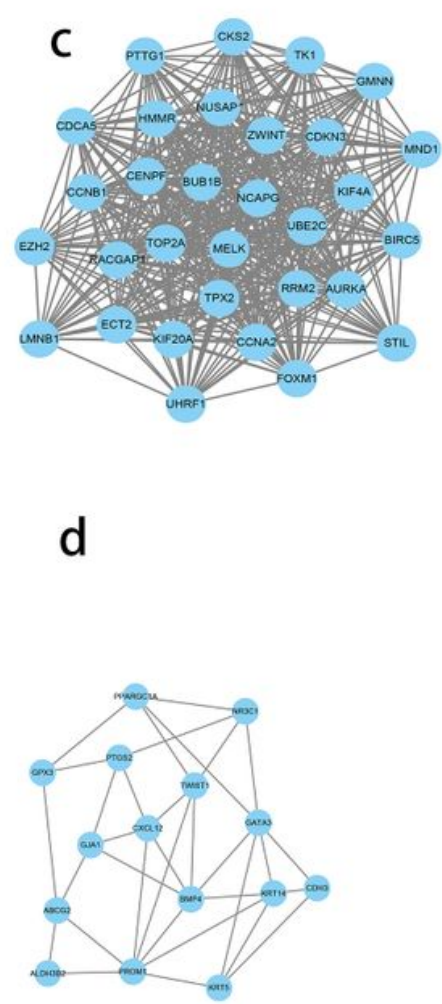
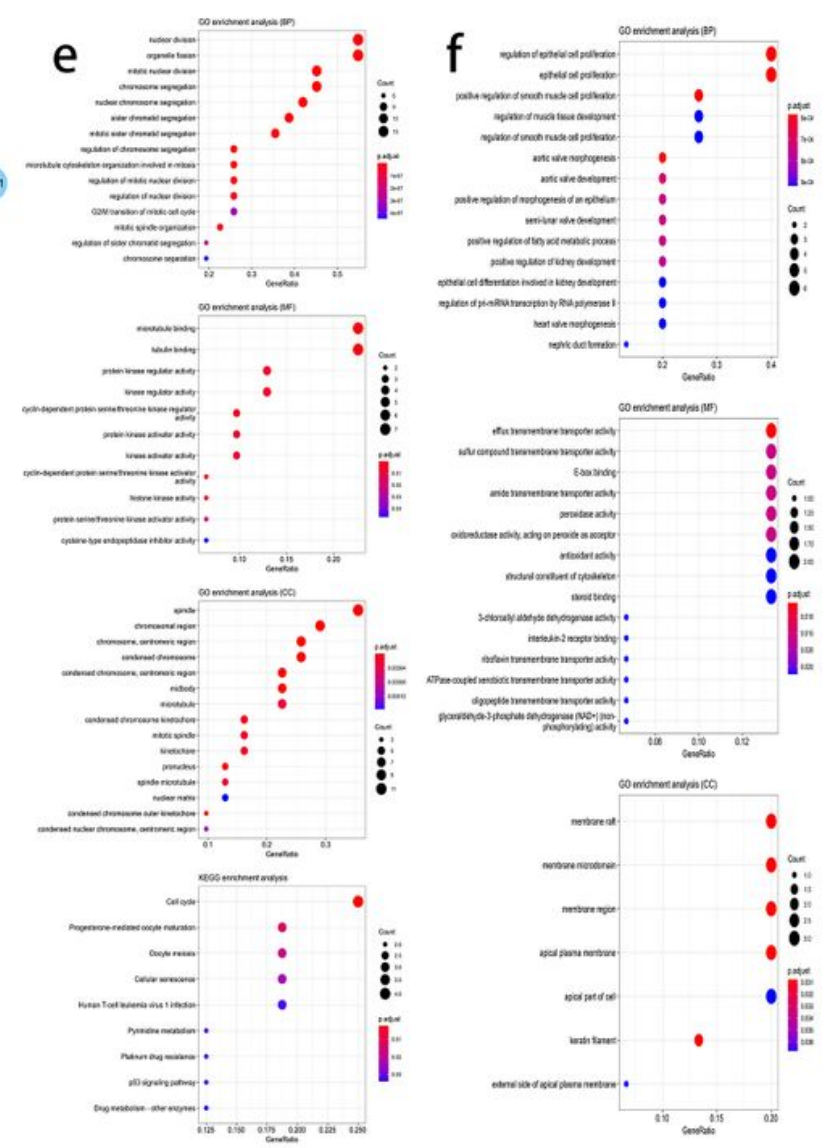

Figure 3

(a) PPI network map of DEGs constructed from the String database. (b) 30 genes with the highest scores of protein interaction calculated using Cytoscape software. (c) Module 1: module with the highest score. (d) Module 2: the second-ranked module. (e) GO and KEGG enrichment analysis of Module 1. (f) GO and KEGG enrichment analysis of Module 2. 

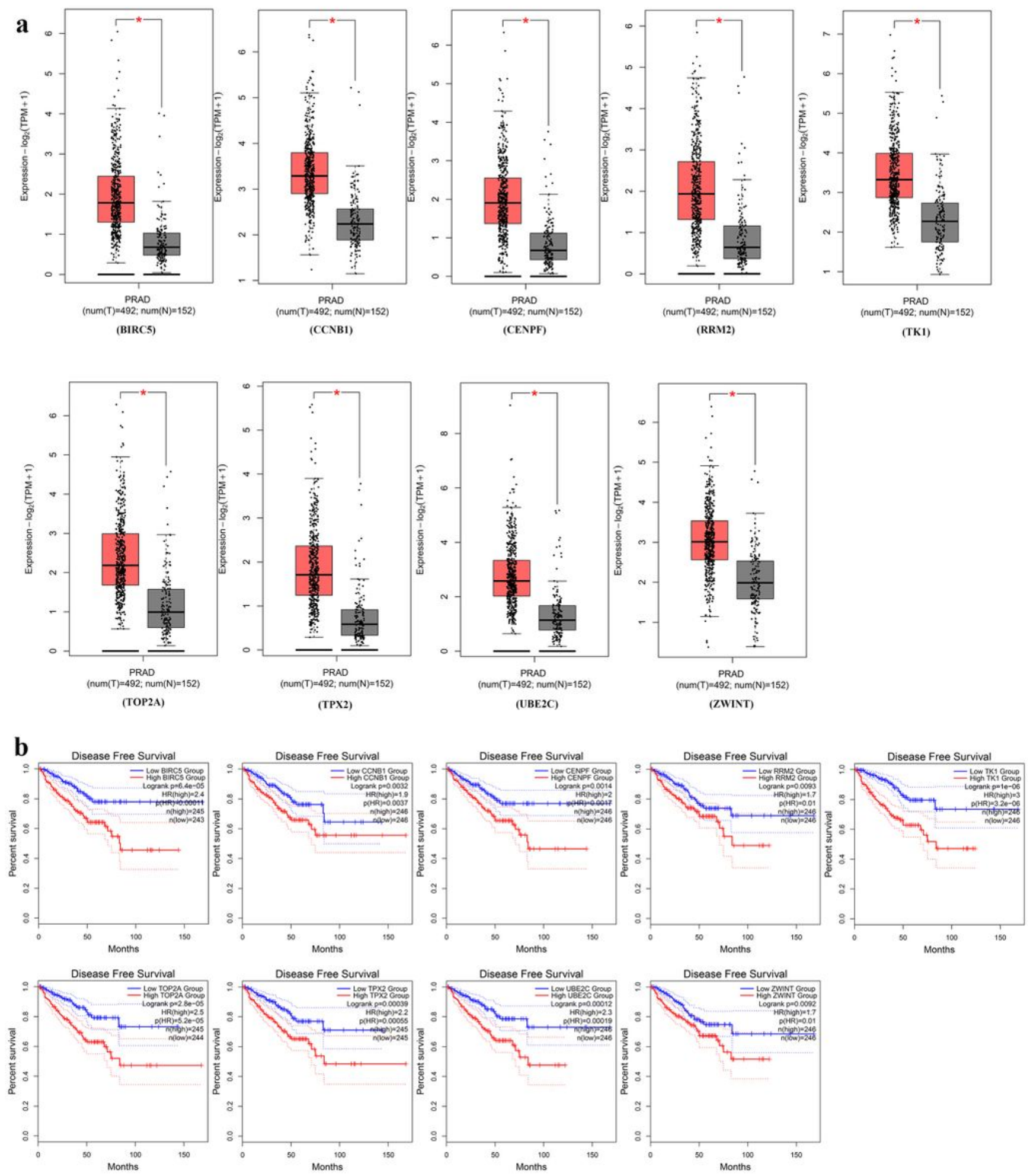

\section{Figure 4}

Results of validation and survival analysis for 30 genes. (a) With $|\log F C|>1$ and $p<0.01$ as the cutoff value, 30 genes are verified in the GEPIA database. (b) Survival analysis results of 30 genes in the GEPIA database. 


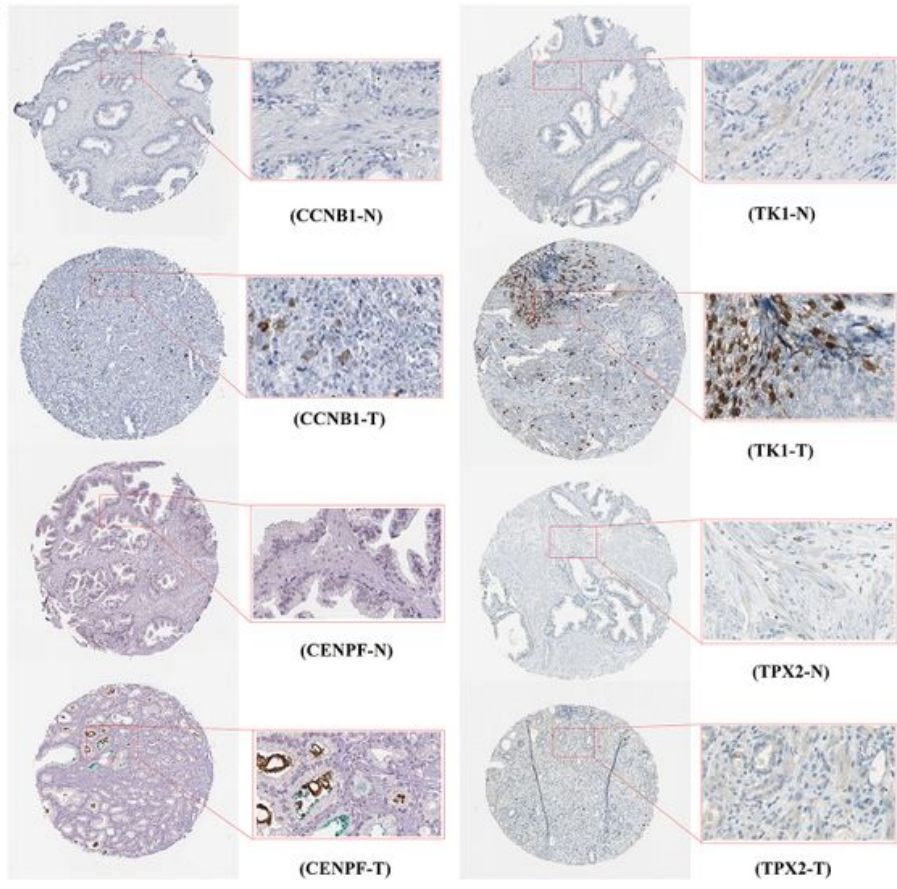

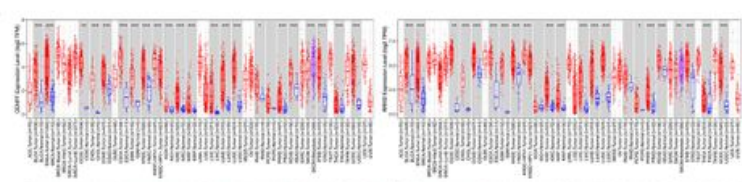
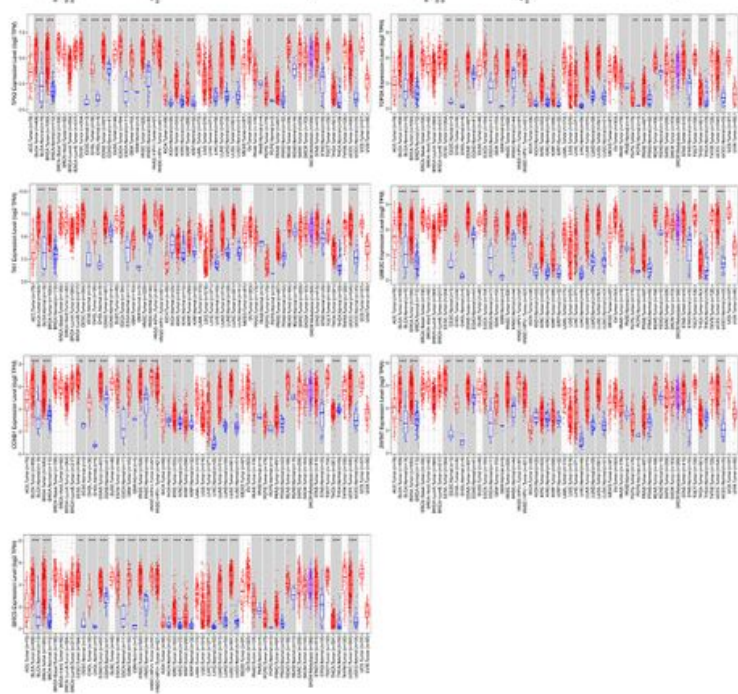

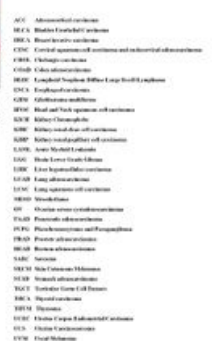

Figure 5

(a) HPA database verification results of 4 key genes,including CCNB1, CENPF, TK1,TPX2. (b) The pancancer analysis results of 9 key genes in the Timer2 database. Red box represents the tumor group, blue box represents the normal group, and*: $p$-value $<0.05$; $* *$ : $p$-value $<0.01$; $* \star \star$ : $p$-value $<0.001$. 


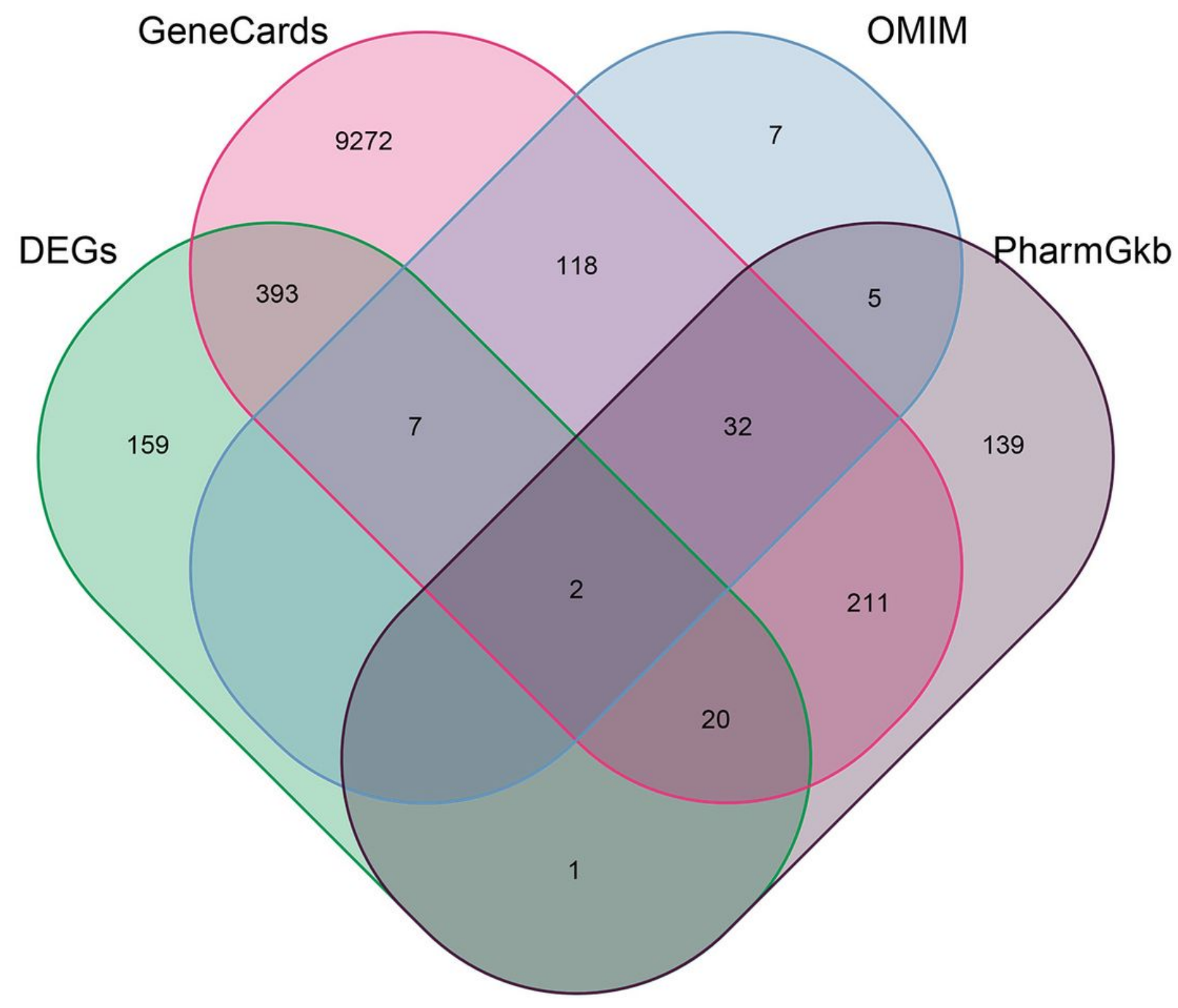

Figure 6

Concatenation of PCa targets from the GeneCards, OMIM, PharmGkb databases and DEGs. 

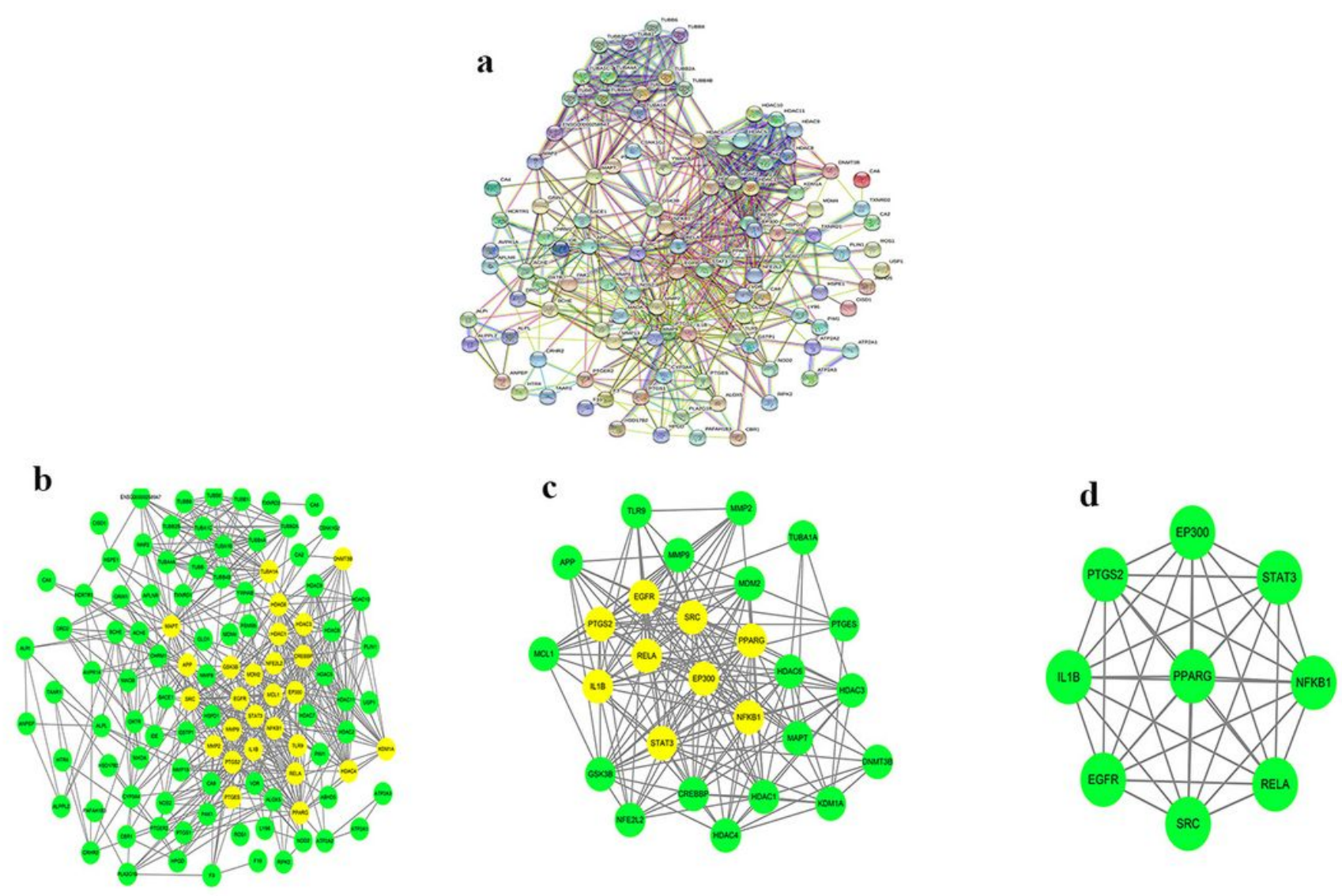

Figure 7

PPI network diagram of PCa-curcumin targets and the screening process. (a) PPI network diagram of PCa-curcumin targets. (b) The yellow part of the diagram shows the groups after the first screening by Cytoscape. (c) Substrates after the first screening. The yellow part of the diagram shows the groups after the second screening. (d) Second screened moiety. 

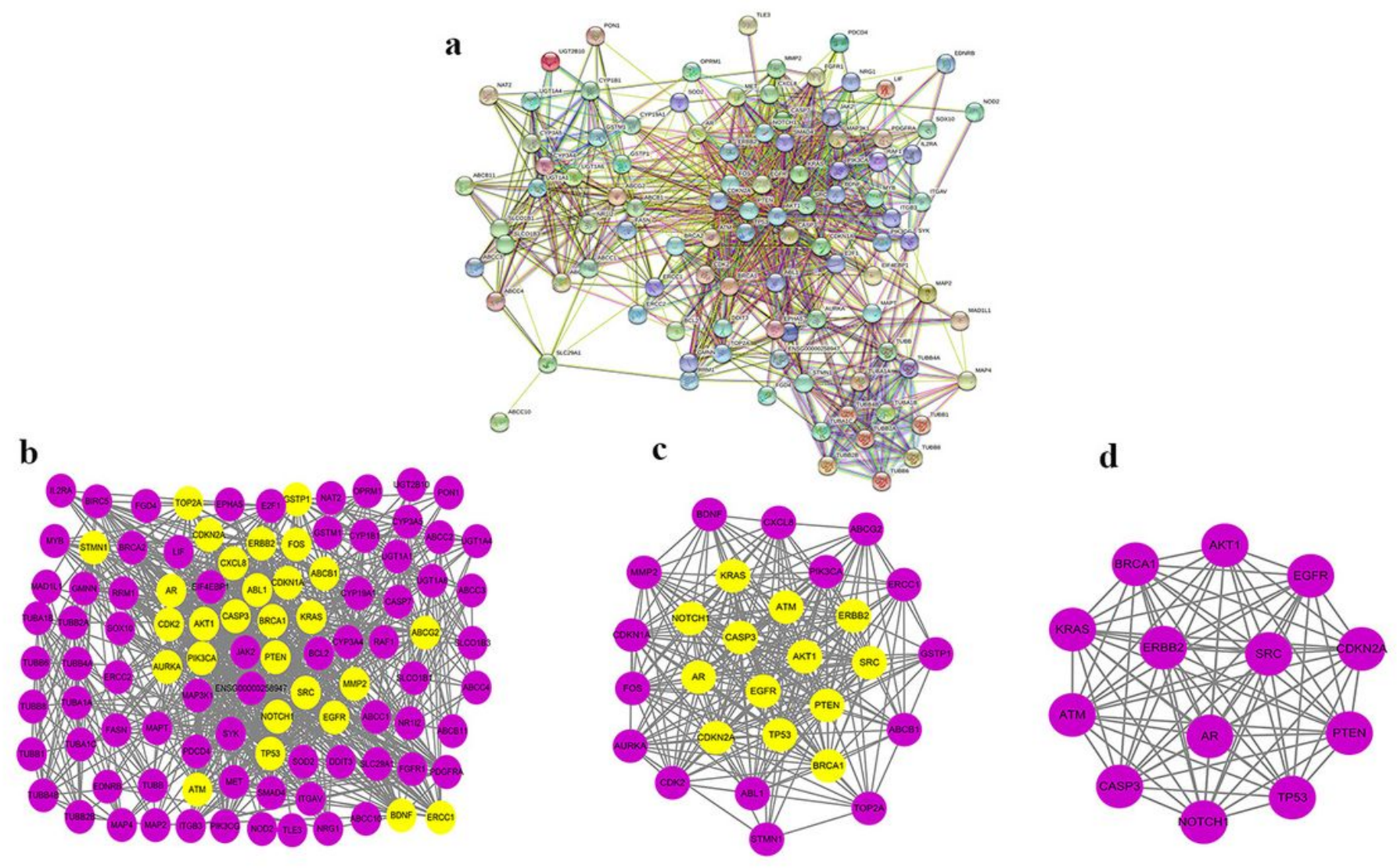

Figure 8

PPI network diagram of PCa-paclitaxel targets and the screening process. (a) PPI network diagram of PCa-paclitaxel targets. (b) The yellow part of the diagram shows the groups after the first screening by Cytoscape. (c) Substrates after the first screening. The yellow part of the diagram shows the groups after the second screening. (d) Second screened moiety. 

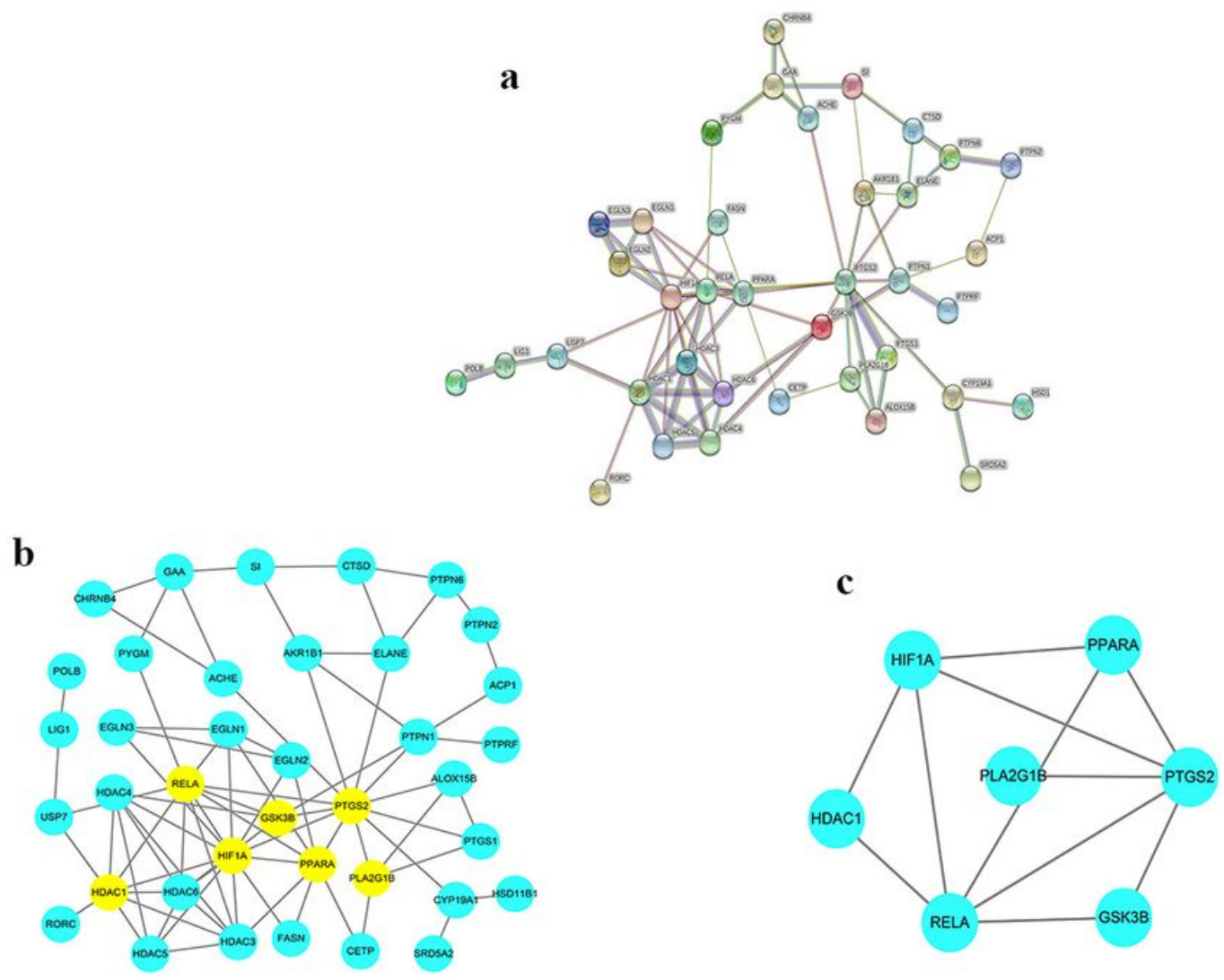

Figure 9

PPI network diagram of PCa-ursolic acid targets and the screening process. (a) PPI network diagram of PCa-ursolic acid targets. (b) The yellow part of the diagram shows the groups after screening. (c) Substrates after screening. 

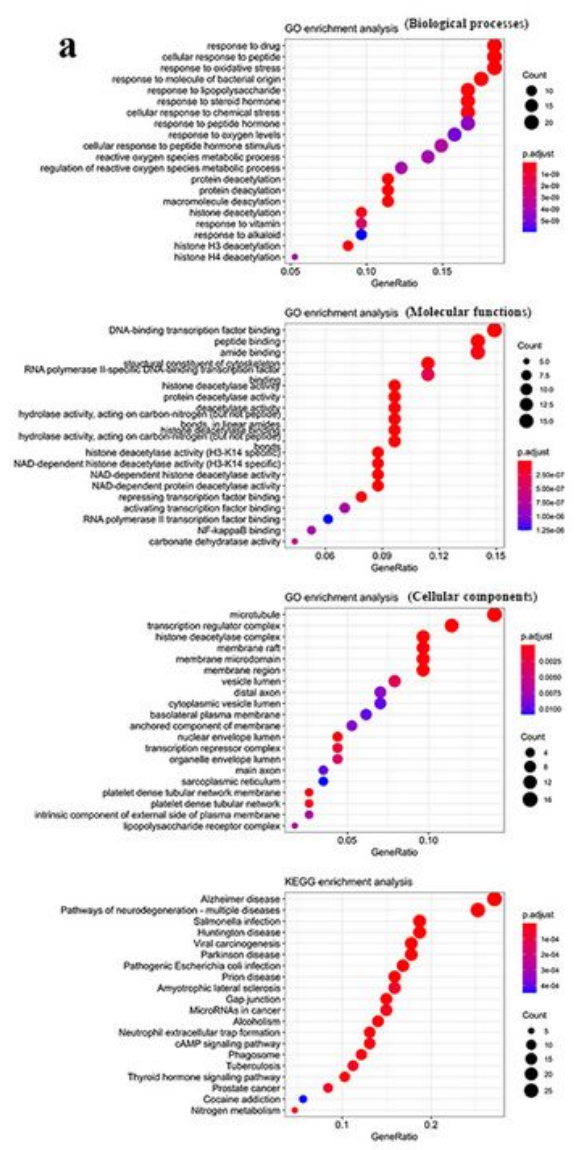
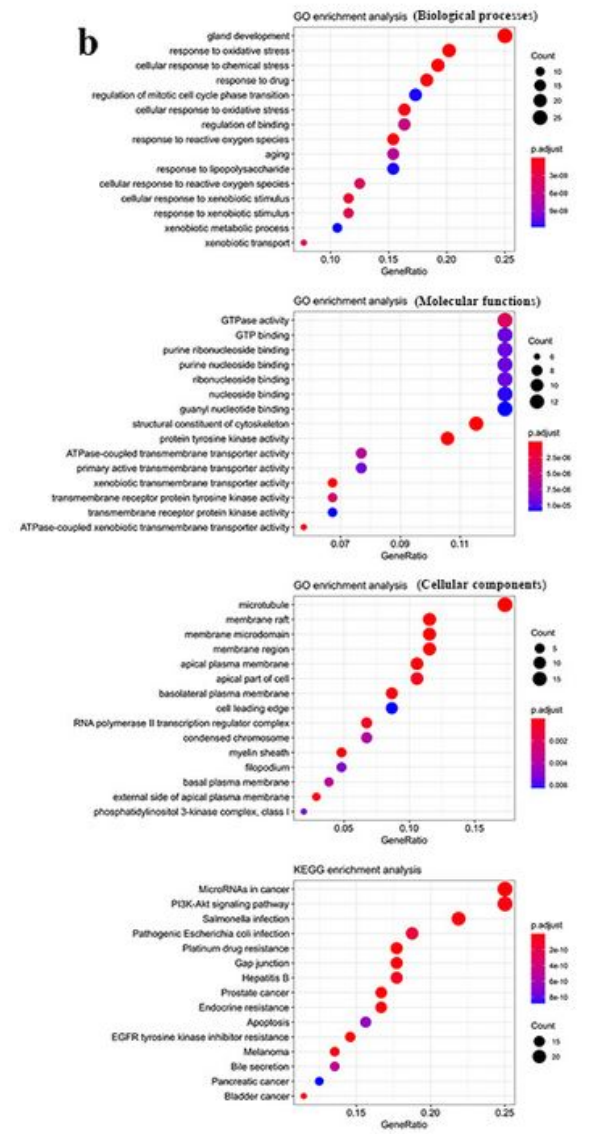
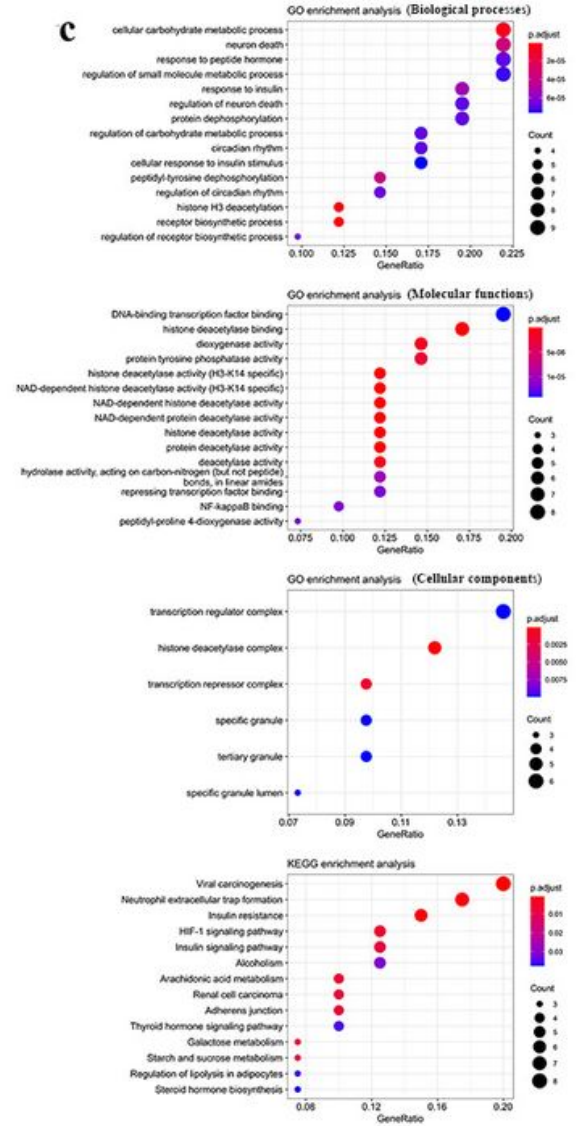

Figure 10

GO, KEGG enriched bubble charts of PCa-natural products. (a) Charts of PCa-curcumin targets. (b) Charts of PCa-paclitaxel targets. (c) Charts of PCa-paclitaxel ursolic acid targets. 
a

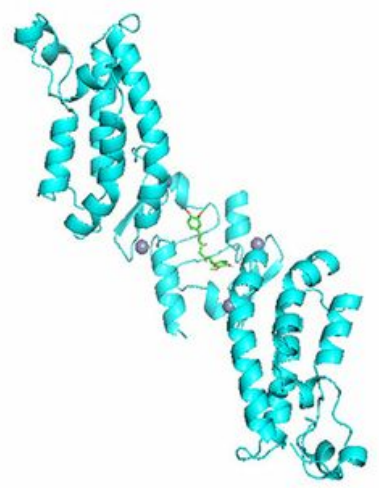

c

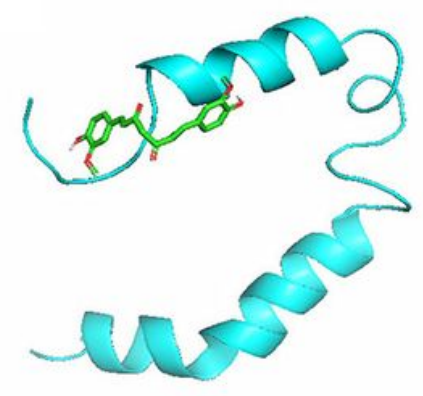

b
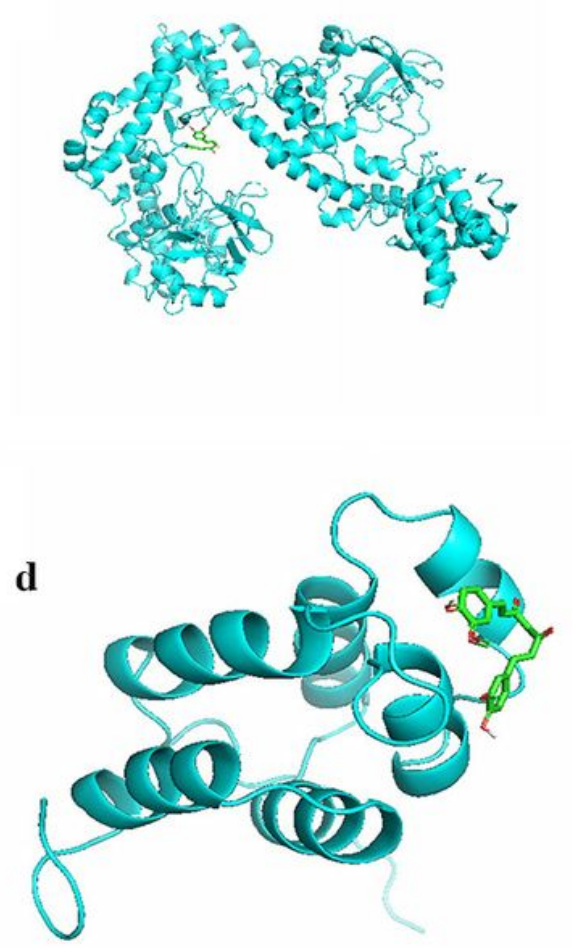

Figure 11

Curcumin was used as the small-molecule ligand and the target as the macromolecular receptor for molecular docking models. (a) Molecular docking model of curcumin and EP300. (b) Molecular docking model of curcumin and RELA. (c) Molecular docking model of curcumin and EGFR. (d) Molecular docking model of curcumin and NFKB1. 

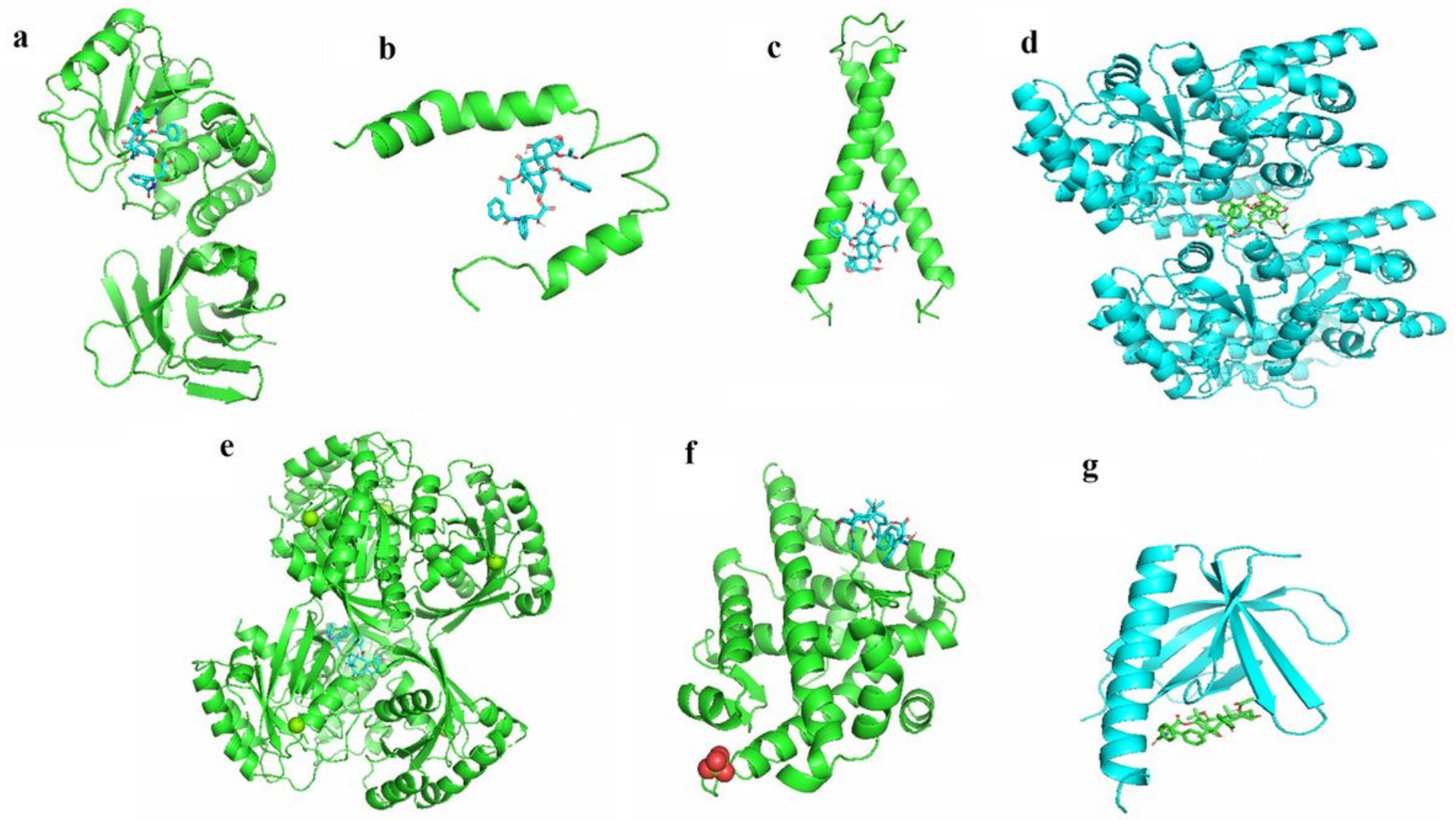

g

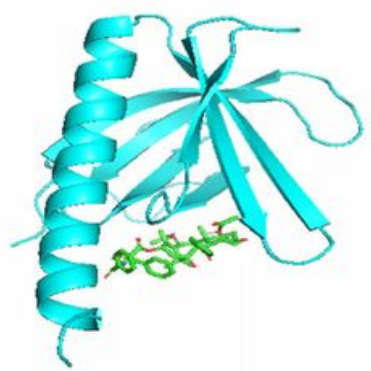

\section{Figure 12}

paclitaxel was used as the small-molecule ligand and the target as the macromolecular receptor for molecular docking models. (a) Molecular docking model of paclitaxel and PTEN. (b) Molecular docking model of paclitaxel and EGFR. (c) Molecular docking model of paclitaxel and ERBB2. (d) Molecular docking model of paclitaxel and TP53. (e) Molecular docking model of paclitaxel and KRAS. (f) Molecular docking model of paclitaxel and AR. (g) Molecular docking model of paclitaxel and AKT1. 


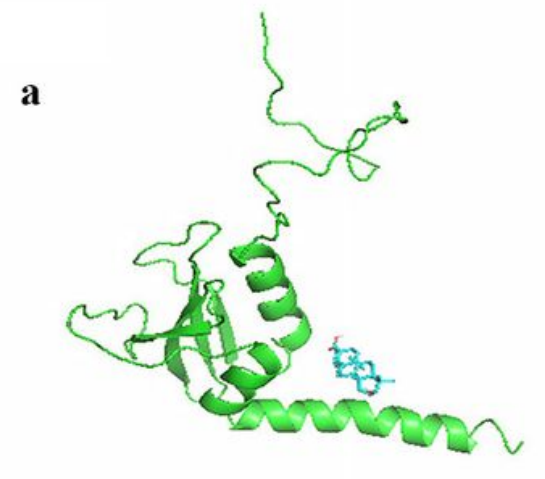

b

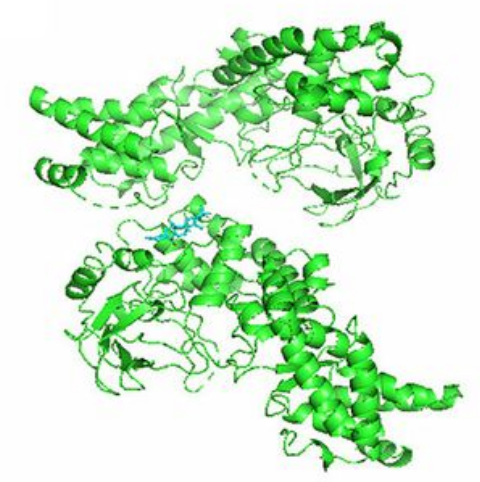

\section{Figure 13}

Ursolic acid was used as the small-molecule ligand and the target as the macromolecular receptor for molecular docking models. (a) Molecular docking model of ursolic acid and GSK3B. (b) Molecular docking model of ursolic acid and RELA.

\section{Supplementary Files}

This is a list of supplementary files associated with this preprint. Click to download.

- Table2.docx

- Additionalfile.docx 